\title{
Discovery of a protein uptake pathway in lysosomes
}

One Sentence Summary: Discovery of a novel proteolytic pathway in cells, the dysfunction of which leads to protein aggregation disease in humans.

Authors: Yuuki Fujiwara ${ }^{1, \dagger}$, Viorica Raluca Contu ${ }^{1, \dagger}$, Chihana Kabuta ${ }^{1, \dagger}$, Megumu Ogawa ${ }^{2}$, Hiromi Fujita ${ }^{1}$, Hisae Kikuchi ${ }^{1}$, Ryohei Sakai ${ }^{1}$, Katsunori Hase ${ }^{1}$, Mari Suzuki ${ }^{3}$, Ikuko Koyama-Honda $^{4}$, Michio Inoue ${ }^{2,5}$, Yasushi Oya ${ }^{6}$, Yukiko U. Inoue ${ }^{7}$, Takayoshi Inoue ${ }^{7}$, Ryosuke Takahashi $^{8}$, Ichizo Nishino ${ }^{2,5}$, Keiji Wada $^{1}$, Satoru Noguchi ${ }^{2,5^{*}}$, Tomohiro Kabuta $^{1 *}$

\section{Affiliations:}

${ }^{1}$ Department of Degenerative Neurological Diseases, National Institute of Neuroscience, National Center of Neurology and Psychiatry, Tokyo, Japan.

${ }^{2}$ Department of Neuromuscular Research, National Institute of Neuroscience, National Center of Neurology and Psychiatry, Tokyo, Japan.

${ }^{3}$ Department of Sensory and Motor Systems, Tokyo Metropolitan Institute of Medical Science, Tokyo, Japan.

${ }^{4}$ Department of Biochemistry and Molecular Biology, Graduate School and Faculty of Medicine, The University of Tokyo, Tokyo, Japan.

${ }^{5}$ Medical Genome Center, National Center of Neurology and Psychiatry, Tokyo, Japan. 
${ }^{7}$ Department of Biochemistry and Cellular Biology, National Institute of Neuroscience, National Center of Neurology and Psychiatry, Tokyo, Japan.

${ }^{8}$ Department of Neurology, Graduate School of Medicine, Kyoto University, Kyoto, Japan.

*Correspondence to: noguchi@ncnp.go.jp; kabuta@ncnp.go.jp

$5 \quad \dagger$ These authors contributed equally to this work.

\begin{abstract}
The degradation of cellular components plays an essential role in homeostasis. However, the known degradation pathways cannot account for the levels of proteolysis in cells. Here, we demonstrate that cytosolic proteins are imported into lysosomes in an ATP-dependent 10 manner for degradation through a direct uptake mechanism distinct from any known pathway. SIDT2, a lysosomal membrane protein previously reported as an RNA transporter, translocates substrate proteins across the lysosomal membrane. Furthermore, we identify a dominant-negative mutation in SIDT2 that causes neuropathy and distal myopathy with rimmed vacuoles, a protein aggregation disease in humans. We generate Sidt2 knockout mice, recapitulating the characteristic features of this disease. Our results reveal a novel degradation pathway and illustrate its crucial role in cellular proteostasis, physiology, and pathophysiology.
\end{abstract}

Biological homeostasis is dependent on cyclic synthesis and degradation of cellular components. As such, regulated degradation of biomacromolecules is essential for maintaining the intracellular environment, with dysfunction in degradation pathways associated with various diseases. Lysosomes are the principal organelles involved in the degradation of macromolecules. The cytosolic components are delivered into lysosomes and degraded during the process of autophagy 
$(1,2)$. Various cellular components are processed via distinct autophagic pathways $(1,2)$, including macroautophagy, microautophagy, and chaperone-mediated autophagy (CMA) (Supplementary text A). Previously, we reported the direct uptake of nucleic acids by lysosomes via RNautophagy and DNautophagy (RDA) (3, 4). Although the underlying mechanism and physiological roles of macroautophagy have been extensively studied, research on non-macroautophagic pathways and their contributions is limited.

\section{A novel ATP-dependent autophagic pathway for protein degradation}

Major intracellular degradation pathways identified to date can account for only a portion of the total proteolysis within cells $(5,6)$. Knockdown of Atg5, a gene required for macroautophagy, did not have a significant effect on the basal level of protein degradation in mouse embryonic fibroblasts (MEFs) (Fig. S1, A and B; Supplementary text B). However, treatment with two inhibitors of lysosomal acid proteases, pepstatin A and E64d (Pep+E64), markedly decreased total proteolysis levels in $A t g 5^{--}$MEFs (Fig. S1C). Thus, non-macroautophagic lysosomal pathways play an important role in basal protein degradation.

To investigate the possibility of direct uptake and degradation of protein (Supplementary text C), we developed an in vitro reconstruction assay using isolated lysosomes $(3,4,7,8)$, using $\alpha$-synuclein and tau proteins as substrates. Lysosomes were co-incubated with substrate proteins ( \pm ATP), and the samples were analyzed by western blot and immunoelectron microscopy (Fig. 1A). In the presence of ATP, $\alpha$-synuclein and tau were directly degraded by intact lysosomes (Fig. 1, B and C and Fig. S2, A and B). However, degradation was inhibited following preincubation with lysosomal acid protease inhibitors (Fig. 1D). Using immunoelectron microscopy, we observed that $\alpha$-synuclein protein was directly taken up into the lysosomal lumen (Fig. 1E and Fig. $\mathrm{S} 3$, A to $\mathrm{C}$ ). The removal of protein from the assay solution (corresponding to the import of 
substrate protein into the lysosomal lumen) was ATP-dependent (Fig. S2C; Supplementary text D). This led us to hypothesize that a novel pathway involving ATP-dependent direct uptake of proteins occurs in lysosomes.

\section{Direct uptake of proteins into lysosomes via SIDT2 for subsequent degradation}

E). CMA is a selective pathway for the degradation of cytoplasmic proteins harboring a

KFERQ-like motif, which is bound by the chaperone hsc70 (9). $\alpha$-synuclein protein contains

KFERQ-like motifs, and is known to be a substrate for CMA through these sequences (10).

However, recombinant $\triangle \mathrm{DQ} \alpha$-synuclein which lacks KFERQ-like motifs (10), was directly

degraded by isolated lysosomes in an ATP-dependent manner (Fig. 1F and Fig. S2D).

Furthermore, unlike in CMA, the addition of hsc70 did not have an additive effect on the degradation of $\alpha$-synuclein protein (Fig. 1G and Fig. S2E). Thus, the direct uptake and degradation of protein by isolated lysosomes observed in this study is independent of CMA (Supplementary text F).

In RDA, nucleic acids are directly imported into lysosomes via SIDT2 (2-4, 7, 8, 11), a multi-pass lysosomal membrane protein belonging to the SID-1 nucleic acid transporter family $(12,13)$. While we have previously shown that SIDT2 mediates lysosomal uptake of nucleic acids for subsequent degradation $(2-4,7,8,11)$, its role in proteolysis remains unknown. We isolated lysosomes from Sidt $2^{+/+}$and Sidt $2^{-/-}$MEFs and compared protein degradation levels in the presence of ATP. Protein degradation was attenuated in isolated lysosomes derived from Sidt $2^{-/-}$ MEFs (Fig. 1H), while lysosomal $\mathrm{pH}$ and the proteolytic activity of luminal hydrolases were unaltered (Fig. S4, A and B). In addition, protein degradation was increased in lysosomes derived 
from SIDT2-overexpressing cells (Fig. 1I). Together, these results demonstrate direct ATP-dependent protein uptake into lysosomes via SIDT2.

\section{Protein uptake via SIDT2 contributes to lysosomal proteolysis in cells}

We next analyzed the effect of Sidt2 knockdown on degradation of radiolabeled proteins in MEFs. A significant decrease in proteolysis was observed in both $A \operatorname{tg} 5^{-/-}$and $\operatorname{Atg} 5^{+/+}$MEFs after knockdown of Sidt2 (Fig. 2A and Fig. S5, A to D). In contrast, knockdown of Lamp2a (14) and Vps4 $(15,16)$ did not have a significant effect on proteolytic activity (Fig. S5A; Supplementary text G). The suppressive effect of Sidt2 knockdown was not observed in cells treated with Pep+E64, confirming that SIDT2-dependent proteolysis occurs in lysosomes (Fig. 2A and Fig. S5, B and C). Moreover, in Sidt2 siRNA-treated Atg $5^{+/+}$MEFs, the protein degradation levels in Pep+E64d-untreated cells were almost equivalent to those in Pep+E64d-treated cells (Fig. 2A). Thus, SIDT2-dependent proteolysis accounts for a large proportion of the observed lysosomal degradation of intracellular proteins.

Total degradation of intracellular protein was also markedly increased by SIDT2 overexpression (Fig. 2B). The degradation of individual proteins was also examined using the Tet-Off system in neuro2a (N2A) cells and HeLa cells. Here, overexpression of SIDT2 markedly increased intracellular degradation of various cytosolic proteins, including $\alpha$-synuclein and tau (Fig. 2C and D and Fig. S6, A to G; Supplementary text H). Moreover, proteolysis induced by SIDT2 was completely inhibited by Pep+E64 treatment (Fig. 2E). The SIDT2-dependent transport of protein into lysosomes was also observed using $\alpha$-synuclein tagged with fluorescent proteins (17) (Fig. 2F and Fig. S7, A and B). Together, these results indicate that SIDT2-dependent lysosomal translocation and degradation of proteins is a cellular process. Further, SIDT2-dependent 
proteolysis in cells is also independent of all known mechanisms of autophagy (Fig. S8, A to H and

Fig. 2, G to J; Supplementary text I; Fig. S9, A and B).

\section{The mechanism underlying SIDT2-mediated translocation of proteins into lysosomes is similar to the mechanism for nucleic acid transport}

No increase in protein degradation was observed after overexpression of a mutant SIDT2 (18) lacking lysosomal targeting signals (Fig. 3A), indicating that localization of SIDT2 to the lysosomal membrane is required for proteolysis. SIDT2 directly binds substrate nucleic acids via arginine residues in its cytoplasmic loop, and this interaction is necessary for the direct uptake of nucleic acids into lysosomes by SIDT2 (19). The cytoplasmic domain of SIDT2 also directly binds $\alpha$-synuclein protein, and substitution of the aforementioned arginine residues abolishes both the binding and the degradation of $\alpha$-synuclein protein (Fig. 3, B and C). Arginine residues are known to facilitate a wide range of protein-protein interactions through their guanidinium group (20). Together, these results strongly suggest that a direct interaction between SIDT2 and the substrate protein is a necessary step for proteolysis by SIDT2. vitro (Fig. 3D). Considering that the receptor enters the lysosomal lumen via invagination with the cargo during microautophagy, the molecular basis of the novel pathway appears to be different (Supplementary text J). Ablation of the putative hydrolase-active site of SIDT2 (21), which is required for nucleic acid transport $(7,8,22)$, abolished SIDT2-dependent proteolysis (Fig. 3E). Thus, the protein is imported into lysosomes by SIDT2 via a mechanism similar to that observed during RDA. As this pathway is independent of other known mechanisms of autophagy, this is a novel intracellular pathway that directly delivers proteins into the lysosome. Considering our previous findings that lysosomes directly import nucleic acids via a similar mechanism $(2-4,7,8$, 
11), we have identified a general mechanism through which 'diverse cytosolic macromolecules are delivered directly into the lysosome lumen via membrane proteins'. We have named this general mechanism “direct-uptake-via/through-membrane-protein” (DUMP) (Fig. 3F).

\section{Mutations in SIDT2 cause neuropathy and distal myopathy with rimmed vacuoles}

We next investigated whether loss-of-function mutations involving DUMP are associated with protein aggregation diseases in humans (Supplementary text K). We searched our in-house database of whole-exome sequencing data on neuromyopathic patients and found a heterozygous single-base deletion in SIDT2 in one patient with familial neuropathy and distal myopathy (Fig. 4A). This patient was a 64-year-old Japanese man showing dominant inheritance. Clinically, the patient was diagnosed with neuropathy and distal myopathy with rimmed vacuoles (Supplementary text L). Rimmed vacuolar myopathy $(\mathrm{RVM})(23,24)$ is a protein aggregation disease, also known as inclusion body myopathy. RVM is pathologically characterized by the presence in muscle fibers of "rimmed vacuoles", cytosolic depositions surrounded by abnormal vacuoles, which usually contain a variety of aggregate-prone proteins, including phosphorylated tau, $\beta$-amyloid, ubiquitin, and $\alpha$-synuclein (25). Computed tomography and histopathological analyses of the patient biopsy revealed typical features of RVM (Supplementary text M; Fig. 4, B to F). Whole-exome sequencing of gDNA revealed the presence of a heterozygous variant, c.2226delG in SIDT2. This mutation is predicted to cause a frameshift at $743 \mathrm{Arg}$, resulting in the replacement of 90 amino acid residues at the C-terminus (encoding two putative transmembrane regions) with 72 amino acid residues of aberrant sequence (Fig. S10, A and B). No mutations were observed in other genes known to cause RVM.

To determine whether this variant in SIDT2 is pathogenic, we characterized the molecular properties of this mutant SIDT2. First, we observed that this mutant ( $\Delta 2226 \mathrm{G})$ SIDT2 was 
incapable of increasing intracellular proteolysis (Fig. 4G), even though the mutant protein was localized normally to lysosomes (Fig. S10C). In addition, we observed aberrant hypo-glycosylation in $\Delta 2226$ G SIDT2 (Fig. S10D). Surprisingly, glycosylation of wild-type (WT) SIDT2 was generally decreased to a similar level following co-expression with the $\Delta 2226 \mathrm{G}$ mutant (Fig. 4H and Fig. S10D). We also observed that glycosylated (upper band) SIDT2 protein levels were decreased in a muscle biopsy of the patient on immunoblots specific to both WT and $\triangle 2226 \mathrm{G}$ SIDT2, while hypo-glycosylated (lower band) SIDT2 protein levels were increased (Fig. 4, I and J). We confirmed that the WT and the mutant SIDT2 transcripts were expressed at equivalent levels in the patient (Fig. S10A). From these observations, we speculate that $\Delta 2226 \mathrm{G}$ SIDT2 may be pathogenic with a dominant-negative effect. Remarkably, co-expression with $\triangle 2226 \mathrm{G}$ SIDT2 severely impaired the increase in proteolysis induced by WT SIDT2 (Fig. 4K), indicating the dominant-negative effect of $\triangle 2226 \mathrm{G}$ SIDT2 on DUMP activity. Together, these observations strongly suggest that SIDT2-dependent proteolysis is reduced in RVM muscle, providing a convincing explanation for the pathological features seen in the patient. Hence, SIDT2 loss-of-function mutations may cause RVM in humans.

\section{DUMP deficiency resulted in RVM-like phenotypes}

To investigate whether SIDT2 dysfunction induces myopathy, and to elucidate the impact of DUMP in vivo, we generated SIDT2-deficient mice using the CRISPR/Cas9 system (Fig. S11A). Importantly, the SIDT2-deficient mice recapitulated the typical symptoms of RVM observed in the patient (Supplementary text N; Fig. 5, A to H and Fig. S11, B to E). Because diseases with rimmed vacuoles share commonalities in their pathogenesis with impaired protein catabolism and intracellular deposit formation (24), we speculated that deficiency of SIDT2 may cause RVM through the impairment of DUMP (Supplementary text O). We observed that degradation of 
proteins by DUMP was almost completely abolished in lysosomes derived from Sidt $2^{-/-}$muscles (Fig. 5I). RNA degradation by DUMP (RNautophagy) was also attenuated in these lysosomes (Fig. S11F). This is consistent with our observation that various RNAs were also accumulated in the Sidt $2^{-/-}$muscles (Fig. S11G). These findings provide evidence that SIDT2 loss-of-function mutations result in DUMP deficiency, which induces an RVM-like phenotype in vivo (Supplementary text P, Fig. S11, H and I).

\section{Discussion}

Taken together, our data demonstrate that lysosomes directly import proteins into their lumen in a novel autophagic mechanism, and that SIDT2 translocates substrate proteins within this pathway (Fig. S12). Direct transport of protein across membranes via membrane proteins also occurs in mitochondria, endoplasmic reticulum, and peroxisomes (26). In these organelles, transport is mediated via multi-pass membrane proteins that form translocon pores capable of transporting proteins even in a folded state. The precise mechanism by which SIDT2 (a known nucleic acid transporter), mediates the uptake of proteins remains to be clarified. Previous studies on the flexibility of translocon pores in other organelles, as well as other transporters, may help to resolve this issue. In proton-dependent oligopeptide transporters (POTs), arginine residues play a pivotal role in the recognition of substrate peptides (27). POTs are capable of transporting almost all di/tripeptides, and their high poly-specificity is attributed to arginine, lysine, glutamate, and tyrosine residues in their substrate-binding sites (27). All of these residues are also enriched in the cytosolic domain of SIDT2 (Fig. 3B). Additional studies on the crystal structure of SIDT2 (and the role of possible cofactors) are required to resolve this issue. High poly-specificity is also observed 
in transporters of smaller molecules, such as aquaporins, which transport multiple types of molecules that share little similarity in structure (28).

The characteristic features of RVM that are shared between the patient harboring a c.2226delG mutation in SIDT2 and the Sidt2 $2^{-/-}$mouse provide further insight into the physiological and pathophysiological role of DUMP in vivo. RVM is essentially a protein aggregation disease (29), with characteristic aggregates of endogenous proteins at the lesion sites. A previously reported skeletal muscle-specific SIDT2 deficient mouse model presents the pathological characteristics of autophagic vacuolar myopathy (AVM) (30), which is not a protein aggregation disease. In contrast to RVM, the primary cause of AVM is a dysfunction in the luminal region of lysosomes. However, neither luminal pH nor hydrolase activity are affected by SIDT2 deficiency in our studies (Fig.S4, A and B). The contrasting characteristics of the two different SIDT2-deficient mouse models require further clarification. In addition, muscle tissues from mice deficient for macroautophagy (Fig. S11, H and I) and CMA (31) show distinct pathological features compared with SIDT2-deficient muscles (Supplementary text Q). the patient reported in this study. Proteostasis in skeletal muscle under DUMP deficiency may be initially sustained during younger age through other cellular mechanisms (Supplementary text R) $(23,29)$. Our findings provide new insights into the involvement of defects in the translocation system for degradation substrates into lysosomes in the pathomechanism of RVMs. DUMP may be part of the integrated system for proteostasis in postmitotic long-lived tissues, such as muscles and neurons. Such systems are thought to be particularly important for the quality control of cellular proteins during stress conditions, including aging and disease. Therefore, DUMP may 
represent a novel target for therapeutic intervention for degenerative diseases in muscles and neurons.

\section{References and Notes:}

728-741 (2011).

2. W. W. Yim, N. Mizushima, Lysosome biology in autophagy. Cell Discov 6, 6 (2020).

3. Y. Fujiwara et al., Discovery of a novel type of autophagy targeting RNA. Autophagy 9 , 403-409 (2013).

4. Y. Fujiwara et al., Direct uptake and degradation of DNA by lysosomes. Autophagy 9 , 1167-1171 (2013).

5. N. Mizushima et al., Dissection of autophagosome formation using Apg5-deficient mouse embryonic stem cells. J Cell Biol 152, 657-668 (2001).

6. M. Tong, J. M. Smeekens, H. Xiao, R. Wu, Systematic quantification of the dynamics

15 of newly synthesized proteins unveiling their degradation pathways in human cells. Chemical

Science 11, 3557-3568 (2020).

7. S. Aizawa et al., Lysosomal membrane protein SIDT2 mediates the direct uptake of DNA by lysosomes. Autophagy 13, 218-222 (2017).

8. S. Aizawa et al., Lysosomal putative RNA transporter SIDT2 mediates direct uptake of RNA by lysosomes. Autophagy 12, 565-578 (2016).

9. S. Kaushik, A. M. Cuervo, The coming of age of chaperone-mediated autophagy. Nat Rev Mol Cell Biol 19, 365-381 (2018). 
10. A. M. Cuervo, L. Stefanis, R. Fredenburg, P. T. Lansbury, D. Sulzer, Impaired degradation of mutant alpha-synuclein by chaperone-mediated autophagy. Science $\mathbf{3 0 5}$, 1292-1295 (2004).

11. Y. Fujiwara, K. Wada, T. Kabuta, Lysosomal degradation of intracellular nucleic acids-multiple autophagic pathways. J Biochem 161, 145-154 (2017).

12. E. H. Feinberg, C. P. Hunter, Transport of dsRNA into cells by the transmembrane protein SID-1. Science 301, 1545-1547 (2003).

13. G. Jialin, G. Xuefan, Z. Huiwen, SID1 transmembrane family, member 2 (Sidt2): a novel lysosomal membrane protein. Biochem Biophys Res Commun 402, 588-594 (2010). by lysosomes. Science 273, 501-503 (1996).

15. R. Sahu et al., Microautophagy of cytosolic proteins by late endosomes. Dev. Cell 20, 131-139 (2011).

16. J. Mejlvang et al., Starvation induces rapid degradation of selective autophagy receptors by endosomal microautophagy. J Cell Biol 217, 3640-3655 (2018).

17. H. Katayama, A. Yamamoto, N. Mizushima, T. Yoshimori, A. Miyawaki, GFP-like proteins stably accumulate in lysosomes. Cell Struct Funct 33, 1-12 (2008).

18. V. R. Contu et al., Lysosomal targeting of SIDT2 via multiple YxxФ motifs is required for SIDT2 function in the process of RNautophagy. J Cell Sci 130, 2843-2853 (2017).

19. K. Hase et al., Cytosolic domain of SIDT2 carries an arginine-rich motif that binds to RNA/DNA and is important for the direct transport of nucleic acids into lysosomes. Autophagy, $1-15(2020)$. 
20. P. B. Crowley, A. Golovin, Cation-pi interactions in protein-protein interfaces. Proteins 59, 231-239 (2005).

21. J. Pei, D. P. Millay, E. N. Olson, N. V. Grishin, CREST--a large and diverse superfamily of putative transmembrane hydrolases. Biol Direct 6, 37 (2011). 1057-1065 (2011).

23. I. Nishino, Autophagic vacuolar myopathies. Curr Neurol Neurosci Rep 3, 64-69 (2003).

24. I. Nonaka, S. Noguchi, I. Nishino, Distal myopathy with rimmed vacuoles and hereditary inclusion body myopathy. Curr Neurol Neurosci Rep 5, 61-65 (2005).

25. M. C. Malicdan, S. Noguchi, I. Nonaka, Y. K. Hayashi, I. Nishino, A Gne knockout mouse expressing human GNE D176V mutation develops features similar to distal myopathy with rimmed vacuoles or hereditary inclusion body myopathy. Hum Mol Genet 16, 2669-2682 (2007).

26. D. J. Schnell, D. N. Hebert, Protein translocons: multifunctional mediators of protein translocation across membranes. Cell 112, 491-505 (2003).

27. S. Newstead, Towards a structural understanding of drug and peptide transport within the proton-dependent oligopeptide transporter (POT) family. Biochem Soc Trans 39, 1353-1358 (2011). Life Sci 64, 2413-2421 (2007).

29. H. H. Goebel, H. D. Muller, Protein aggregate myopathies. Semin Pediatr Neurol 13, 96-103 (2006). 
30. H. Liu et al., Skeletal muscle-specific Sidt2 knockout in mice induced muscular dystrophy-like phenotype. Metabolism 85, 259-270 (2018).

31. Y. Tanaka et al., Accumulation of autophagic vacuoles and cardiomyopathy in LAMP-2-deficient mice. Nature 406, 902-906 (2000).

Acknowledgments: We thank N. Mizushima for constructive advice and suggestions that improved our work, and for providing $\operatorname{Atg} 5^{-/-}$and $\operatorname{Atg} 5^{+/+}$MEFs, M. Komatsu for providing Atg7-flox mice, K Koishi for providing MuCre-A mice, and M. Shikama and Y Hara for technical assistance. We also thank Editage and Edanz Group for editing the English text of a draft of this manuscript. Funding: This work was supported by a grant from the Japan Agency for Medical Research and Development (AMED) (JP20dm0107127 to T.K.), Grants-in-Aid for Scientific Research from the Japan Society for the Promotion of Science (16H05146, 16H01211 and 19H05710 to T.K. and 17K07124 to K.W.), Grants-in-Aid for JSPS Research Fellows (17J10610 to Y.F. and 15J06173 to V.R.C.), a Grant-in-Aid for JSPS International Research Fellows (18F18384 to V.R.C.), research grants from Takeda Science Foundation (to Y.F. and T.K.), Intramural Research Grants for Neurological and Psychiatric Disorders (30-5 and 30-9 to T.K., 30-9 and 2-6 to S.N., 2-5 to I.N., and 27-9 to K.W.) from the National Center of Neurology and Psychiatry (Japan), and AMED under Grant Number JP20ek0109490h0001 to I.N. and S.N.; Author contributions: Conceptualization: Y.F., S.N., T.K.; Data curation: Y.F., S.N., T.K.;

Formal analysis: Y.F., V.R.C., C.K., M.O., S.N., T.K.; Funding acquisition: Y.F., V.R.C., I.N., K.W., S.N., T.K.; Investigation: Y.F., V.R.C., C.K., M.O., H.F., H.K., R.S., K.H., M.T., M.S., I.K.-H., M.I., Y.O., Y.I.-U., T.I.; Methodology: Y.F., V.R.C., R.S., K.H., I.K.-H., K.W., S.N., T.K.; Project administration: Y.F., S.N., T.K.; Supervision: I.N., S.N., T.K.; Validation: Y.F., 
V.R.C., C.K., S.N., T.K.; Visualization: Y.F., M.O., S.N., T.K.; Writing - original draft: Y.F. and S.N.; and Writing - review and editing: Y.F., S.N., T.K.; Competing interests: The authors declare no competing interests.; and Data and materials availability: All data generated and used in this study are either included in this article or are available from the corresponding authors upon reasonable request. Correspondence and requests for materials should be addressed to T.K. or S.N.

\section{Supplementary Materials:}

Materials and Methods

Supplementary text A - R

Figures S1 - S12 
A

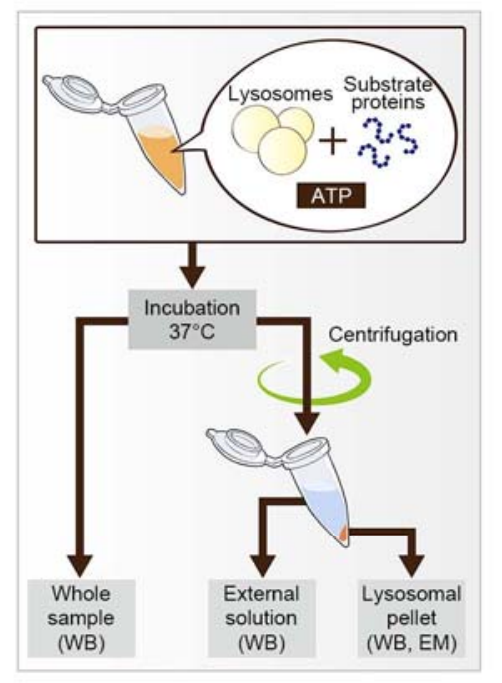

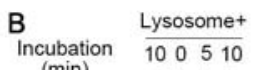
(min)

a-syn

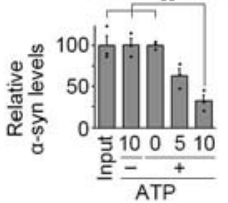

D

Lysosome+
ATP $^{+}$
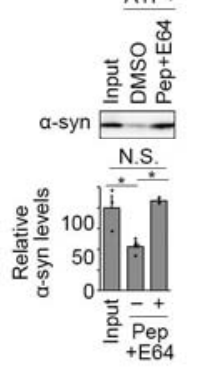

F

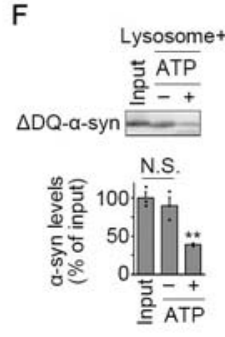

G Lysosome+

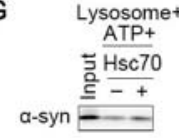

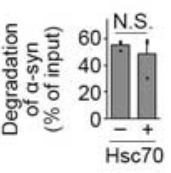

$\mathrm{H}$
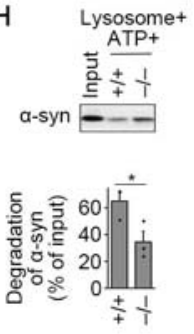

Incubation Lysosome+

(min)

Tau $\stackrel{5}{\rightarrow-1}$

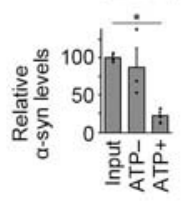

E

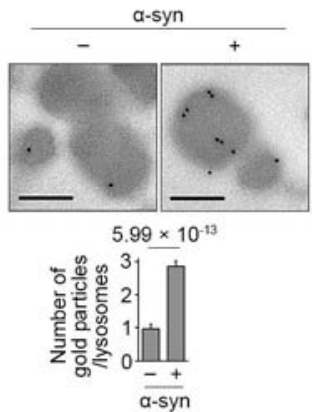

I Lysosome+

$\frac{\text { ATP+ }}{2}$

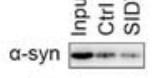

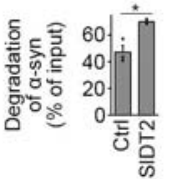

Fig. 1. Lysosomal proteolysis through direct uptake of protein into lysosomes via SIDT2. (A)

Schematic of in vitro reconstruction assay using isolated lysosomes. (B and $\mathbf{C}$ ) Degradation of $\alpha$-synuclein (B) and tau (C) protein by isolated lysosomes derived from $A \operatorname{tg} 5^{+/+}$MEFs (n = 3). (D) Degradation of $\alpha$-synuclein protein by isolated lysosomes derived from $\mathrm{Atg} 5^{+/+} \mathrm{MEFs}$ preincubated with or without Pep+E64. (E) Immunoelectron microscopy image of $\alpha$-synuclein protein in isolated lysosomes co-incubated with or without recombinant $\alpha$-synuclein protein in the presence of ATP. Bars, $200 \mathrm{~nm}$. (F) Degradation of recombinant mutant $\alpha$-synuclein protein that lacks the KFERQ-like motif by isolated lysosomes $(\mathrm{n}=3)$. $(\mathbf{G})$ Degradation of recombinant WT $\alpha$-synuclein protein by isolated lysosomes with or without Hsc70 chaperone $(\mathrm{n}=3)$. $(\mathbf{H}$ and $\mathbf{I})$ 
bioRxiv preprint doi: https://doi.org/10.1101/2020.08.11.245688; this version posted January 26, 2021. The copyright holder for this preprint (which was not certified by peer review) is the author/funder. All rights reserved. No reuse allowed without permission.

Degradation of $\alpha$-synuclein protein by isolated lysosomes derived from SIDT2 $^{+/+}$and SIDT2 ${ }^{-/-}$ MEFs (H) or from SIDT2-overexpressing N2A cells (I). 

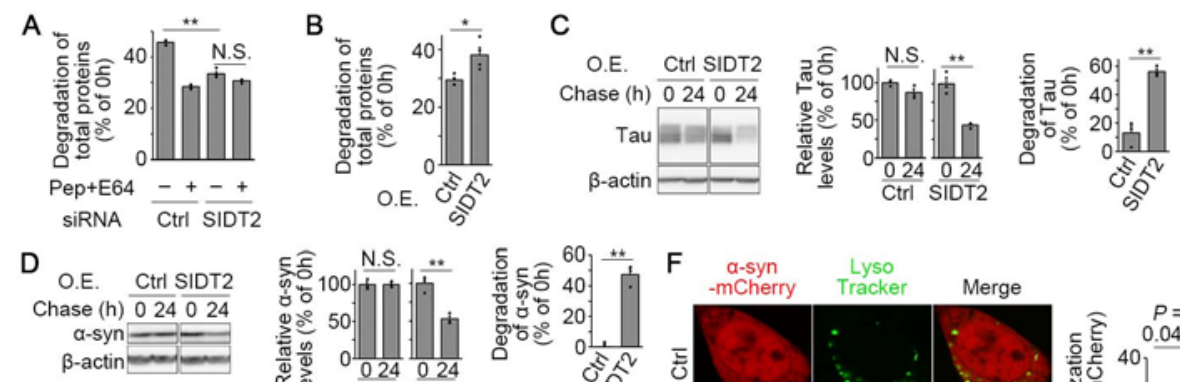

O.E.
Pep+E64 $\frac{\text { SIDT2 }}{-\quad+}$

Chase (h) $\overline{024} \frac{+}{024}$ $\alpha$-syn $-\square$

G O.E. CtrI SIDT2
Chase (h)
$\Delta$ DQ-a-syn
$\beta$-actin B-actin
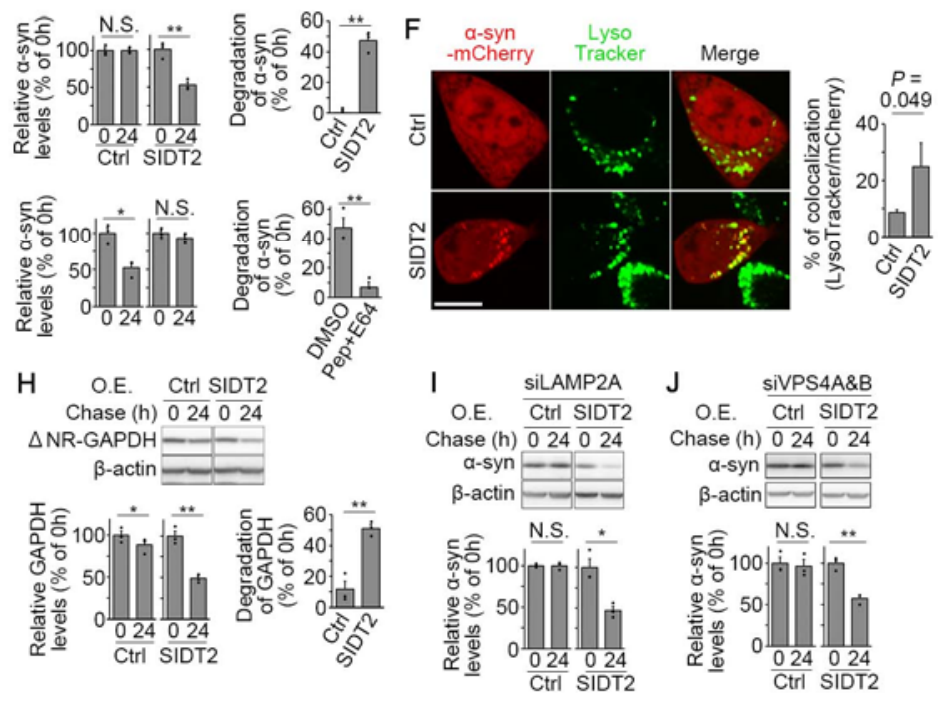

\section{Fig. 2. SIDT2 mediates lysosomal translocation and degradation of proteins in cells. (A)}

Effect of Sidt2 knockdown and lysosomal protease inhibitors on the degradation of radiolabeled total protein in $\operatorname{Atg} 5^{+/+}$MEFs $(n=4)$. (B) Effect of SIDT2 overexpression on the degradation of radiolabeled total protein in N2A cells $(n=4)$. O.E.: overexpression. $(\mathbf{C}$ and $\mathbf{D})$ Degradation of tau (C) and $\alpha$-synuclein (D) protein in N2A cells overexpressing SIDT2, using the Tet-Off system $(\mathrm{n}=$ 3). (E) Degradation of $\alpha$-synuclein protein in SIDT2-overexpressing N2A cells incubated with or without lysosomal protease inhibitors $(n=3)$. (F) Localization of $\alpha$-synuclein-mCherry protein in SIDT2-overexpressing N2A cells (control $\mathrm{n}=14$, SIDT2 $\mathrm{n}=12)$. Bar, $10 \mu \mathrm{m}$. $(\mathbf{G}$ and $\mathbf{H})$

10 Degradation of mutant $\alpha$-synuclein $(\mathrm{G})$ and GAPDH $(\mathrm{H})$ proteins lacking the KFERQ-like motif in N2A cells overexpressing SIDT2, using the Tet-Off system $(n=3)$. (I and $\mathbf{J})$ Degradation of $\alpha$-synuclein protein in SIDT2-overexpressing N2A cells treated with siRNA against Lamp2a (I) or $\operatorname{Vps} 4 a$ and $b(\mathrm{~J})(\mathrm{n}=3) . * \mathrm{p}<0.05, * * \mathrm{p}<0.001$ 
A
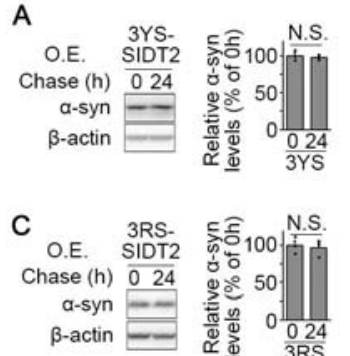

E

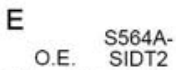

Chase (h) 024

a-syn

$\beta$-actin
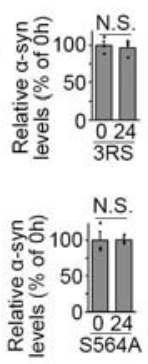

B CWENWRQRKKTLLLAI
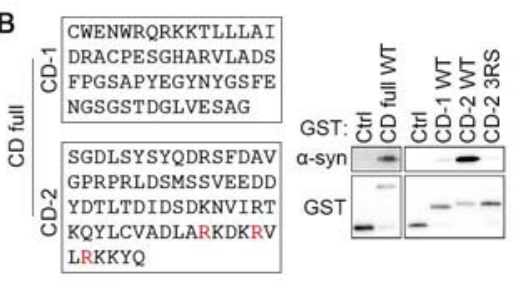

D Lysosome+ (min)
(mito 10

SIDT2
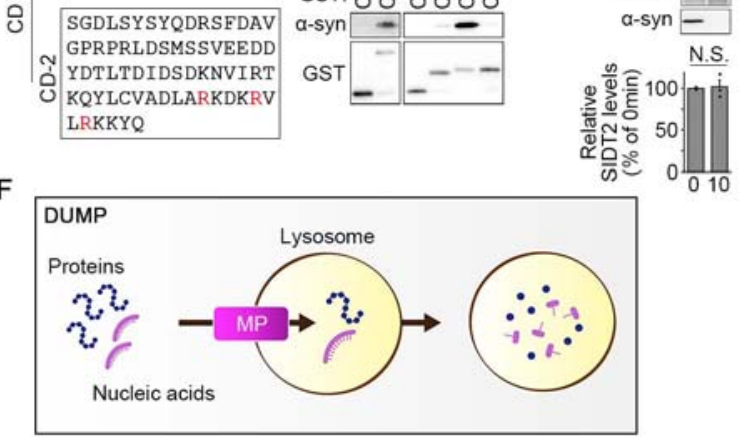

Fig. 3. Mechanism of lysosomal uptake of proteins by SIDT2. (A) Degradation of $\alpha$-synuclein protein in N2A cells overexpressing mutant SIDT2 lacking lysosome targeting signals $(\mathrm{n}=3)$. (B) Interaction of the cytosolic domain of SIDT2 with $\alpha$-synuclein protein. (C) Degradation of $\alpha$-synuclein protein in N2A cells overexpressing mutant SIDT2 that lacks the capacity to bind to $\alpha$-synuclein protein $(\mathrm{n}=3)$. (D) Levels of SIDT2 in isolated lysosomes before and after the incubation $(\mathrm{n}=3)$. ( $\mathbf{E})$ Degradation of $\alpha$-synuclein protein in N2A cells overexpressing mutant SIDT2 lacking a putative hydrolase-active site $(n=3)$. (F) Schematic of DUMP. Proteins and nucleic acids are directly taken up into lysosomes via membrane proteins (MP) and degraded by luminal hydrolases. Lysosomal MP other than SIDT2, and/or yet-unknown cytosolic factors may also be involved in DUMP. ${ }^{*} \mathrm{p}<0.05, * * \mathrm{p}<0.001$. 

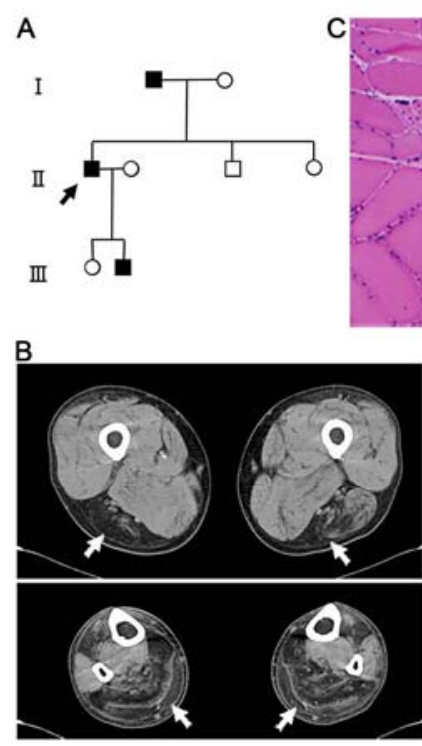

I

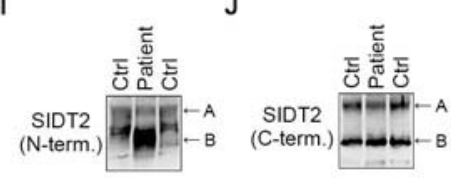

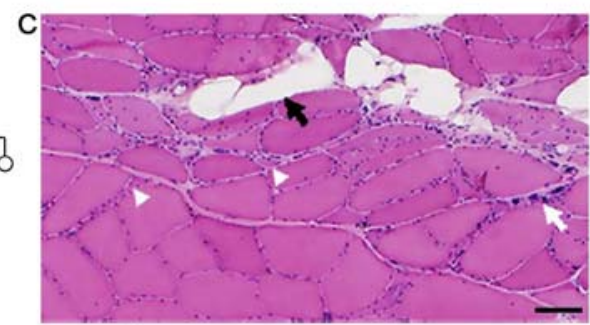

$\mathrm{E}_{\text {LAMP } 2 / \mathrm{p} 62 /}$
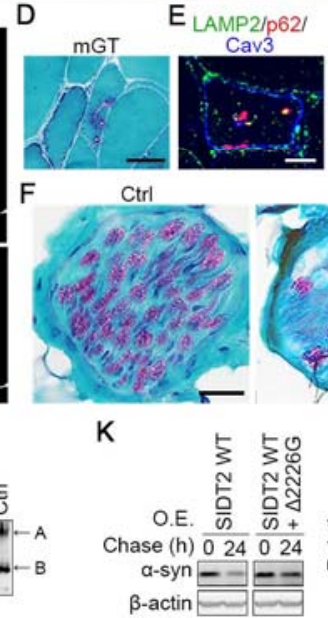

LC $3 / A \beta / C a v 3$

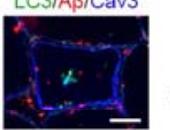

G

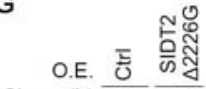

Chase (h) $\overline{024} \overline{024}$

a-syn -actin $=-1$
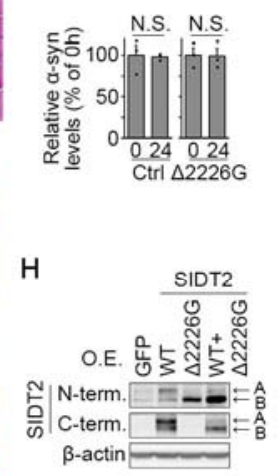

Fig. 4. Loss of function of SIDT2 causes rimmed vacuolar myopathy in humans. (A) Pedigree chart of the proband and family affected by myopathy. The proband is indicated with an arrow. (B) Computed tomography (CT) image of the legs of the patient. The white arrow indicates the lesion. (C) Hematoxylin and eosin (HE)-stained section of skeletal muscle from the patient. The white arrowheads indicate small angular fibers, the white arrow indicates pyknotic nuclear clumps, and the black arrow indicates adipose tissue infiltration. (D) Modified Gomori trichrome (mGT)-stained section of skeletal muscle from the patient showing rimmed vacuoles. (E) Immunohistochemistry of skeletal muscle of the patient. (F) mGT section of peripheral nerve in in cells overexpressing $\Delta 2226 \mathrm{G}$ mutant SIDT2, using the Tet-Off system $(\mathrm{n}=3)$. $(\mathbf{H})$ Immunoblotting of N2A cell lysate overexpressing WT, $\Delta 2226 \mathrm{G}$ mutant, or WT and $\Delta 2226 \mathrm{G}$ mutant SIDT2 together, using specific antibodies against either the $\mathrm{N}$-terminus or the $\mathrm{C}$-terminus 
of SIDT2. (I and $\mathbf{J})$ Immunoblotting of skeletal muscle of the patient using specific antibodies to either the N-terminus (I) or the C-terminus (J) of SIDT2. (K) Degradation of $\alpha$-synuclein protein in cells co-expressing WT and $\Delta 2226 \mathrm{G}$ mutant SIDT2, compared with WT SIDT2 alone $(\mathrm{n}=3)$. Bars represent $40 \mu \mathrm{m} .{ }^{*} \mathrm{p}<0.05, * * \mathrm{p}<0.001$. 

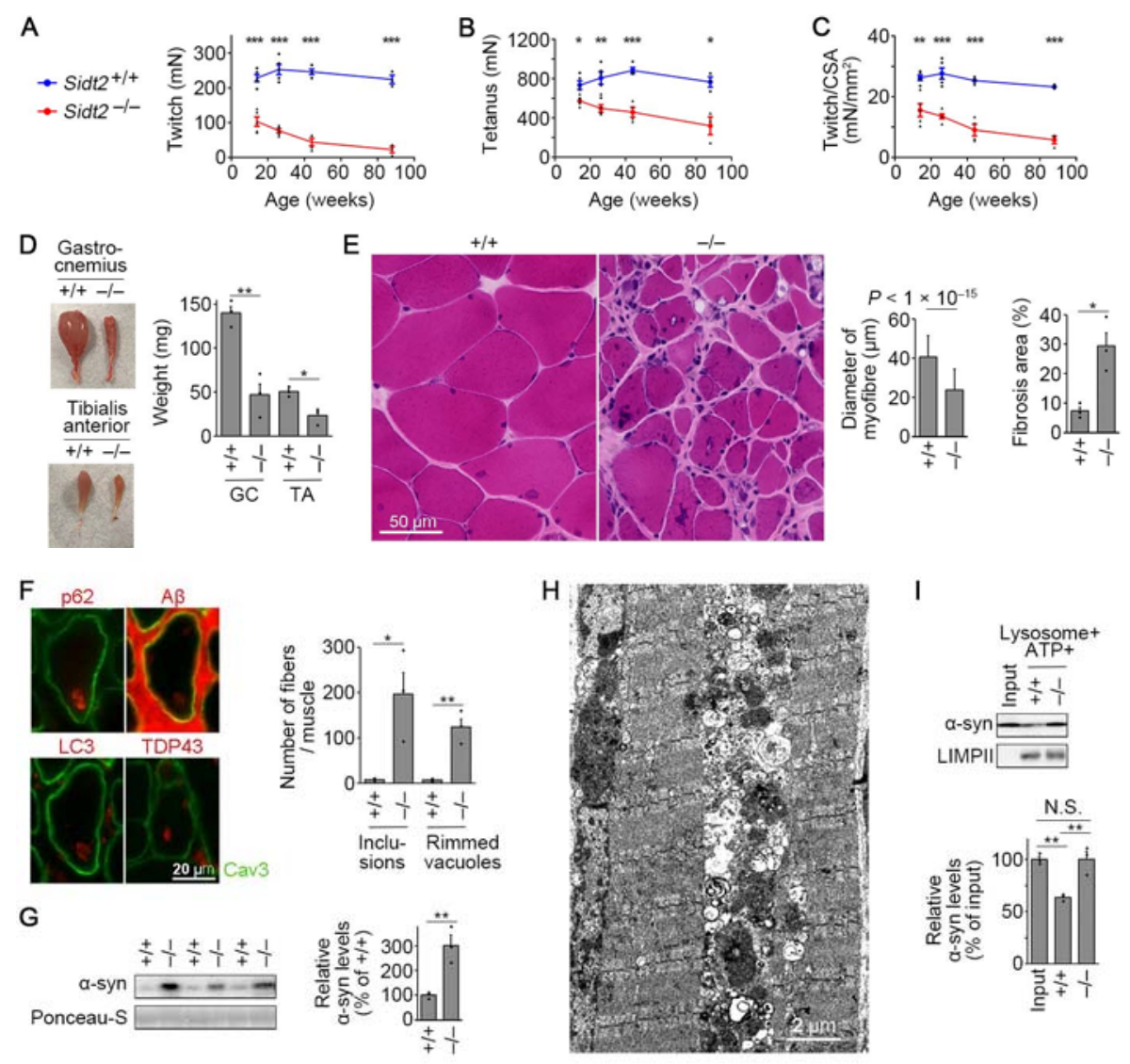

I
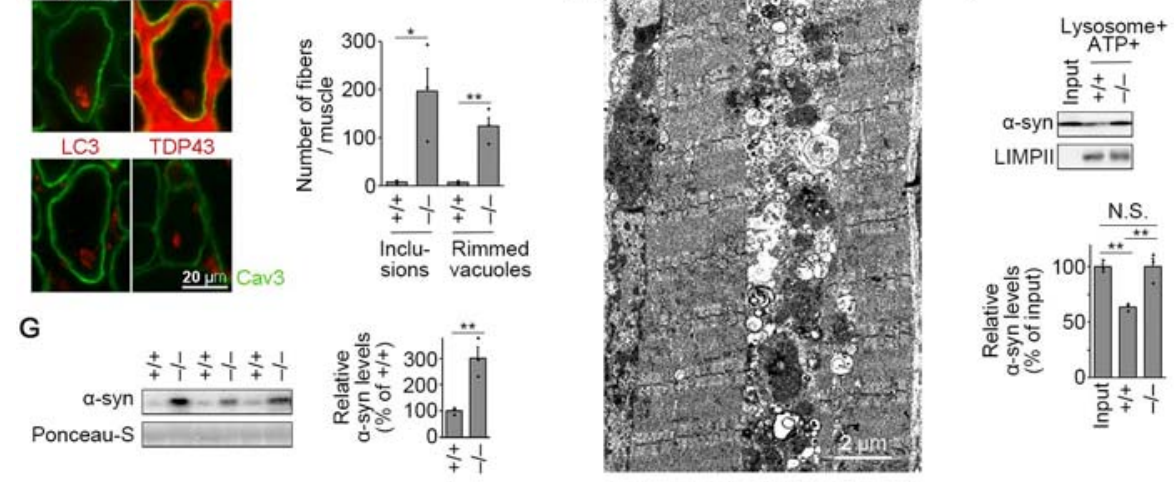

Fig. 5. Sidt2 deficiency abolishes DUMP activity in skeletal muscle and promotes

rimmed-vacuolar myopathic phenotype in mouse. (A to C) Changes in muscle contractions of gastrocnemius in Sidt2 $2^{+/+}$and Sidt $2^{-/-}$mice. Twitch (A), Tetanus (B), and Twitch/SCA (C) (n = 3-5). (D) Skeletal muscles of Sidt2 $2^{+/+}$and Sidt $2^{-/}$mice and their weight $(\mathrm{n}=3)$. (E) HE sections of skeletal muscles of Sidt $2^{+/+}$and Sidt $2^{-/-}$mice and their quantification. Diameter of myofiber, $+/+$: $\mathrm{n}$ $=6,258,-/-: \mathrm{n}=5,350$ from three mice. Fibrosis area, $\mathrm{n}=3$. (F) Immunohistochemistry of skeletal muscles of Sidt $2^{+/+}$and Sidt $2^{-/}$mice $(\mathrm{n}=3)$. (G) Immunoblotting of $\alpha$-synuclein protein in skeletal muscles of Sidt2 ${ }^{+/+}$and Sidt2 ${ }^{-/-}$mice $(\mathrm{n}=3)$. (H) Electron microscopy image of the ultrastructure of cytoplasmic inclusions in skeletal muscle of Sidt $2^{-/-}$mouse. (I) Degradation of $\alpha$-synuclein 
bioRxiv preprint doi: https://doi.org/10.1101/2020.08.11.245688; this version posted January 26, 2021. The copyright holder for this preprint (which was not certified by peer review) is the author/funder. All rights reserved. No reuse allowed without permission.

protein by isolated lysosomes derived from skeletal muscles of WT or Sidt $2^{-/-}$mice $(\mathrm{n}=3)$. * $\mathrm{p}<$

$0.05, * * \mathrm{p}<0.001, * * * \mathrm{p}<0.0001$ 


\section{Supplementary Materials for}

\section{Discovery of a protein uptake pathway in lysosomes}

Authors: Yuuki Fujiwara, Viorica Raluca Contu, Chihana Kabuta, Megumu Ogawa, Hiromi Fujita, Hisae Kikuchi, Ryohei Sakai, Katsunori Hase, Mari Suzuki, Ikuko Koyama-Honda,

Michio Inoue, Yasushi Oya, Yukiko U. Inoue, Takayoshi Inoue, Ryosuke Takahashi, Ichizo

Nishino, Keiji Wada, Satoru Noguchi, Tomohiro Kabuta

Correspondence to: noguchi@ncnp.go.jp; kabuta@ncnp.go.jp

15

\section{This PDF file includes:}

Materials and Methods

Supplementary text A - R

20

Figs. S1 - S12

References (32 - 48) 


\section{Materials and Methods}

siRNAs. siRNAs against Egfp (siCtrl) (targeting sequence: 5'-

CAGCACGACUUCUUCAAGU-3'), mouse Sidt2 (siSIDT2: targeting sequence: 5'-

GAGUUUCCGUCCAGUAUUU-3'), mouse Sidt2 (siSIDT2-2: targeting sequence:

5'-GUACCUCUACCAAAAAGUG-3'), mouse Atg5 (targeting sequence:

5'-GAAAAUGGAUUUCGUUAUA-3'), mouse Lamp2a (targeting sequence:

5'-GACTGCAGTGCAGATGAAG-3'), mouse Vps4a (targeting sequence:

5'-CAACCCUAGCGUUAUGAUU-3'), and mouse Vpa4b (targeting sequence:

5'-CUACCUUGCGAGUGCUACA-3') were purchased from JBioS.

Plasmids. pCI-neo vectors containing Egfp, and WT and mutant forms of mouse Sidt2 and Lamp2a were prepared as described previously $(3,8,18,19)$. cDNA encoding human SIDT2 was subcloned into the vector pCI-neo mammalian expression vector (Promega, E1841). pCI-neo-human $\triangle 2226$ G SIDT2 was generated via mutagenesis using Pfu Turbo DNA Polymerase (Agilent, 600250), in accordance with the manufacturer's instructions. pTRE-Tight vectors harboring each of the FLAG-tagged proteins, pTRE-Tight- $\alpha$-synuclein-mCherry, pTRE-Tight- $\alpha$-synuclein-Egfp-mCherry, pEGFP-N1-Lamp1, and pmCherry-N1-human $\Delta 2226 \mathrm{G}$ SIDT2, were generated by subcloning each construct into pTRE-Tight, pEGFP-N1, or pmCherry-N1 vector, respectively. pTRE-Tight- $\Delta$ NR Gapdh was generated by introducing a mutation into pTRE-Tight-Gapdh by mutagenesis. All resulting plasmid constructs were analyzed by sequencing. 
Cell lines. The $A \operatorname{tg} 5^{-/-}$mouse embryonic fibroblasts (MEFs) and $A \operatorname{tg} 5^{+/+} \mathrm{MEFs}$ were kind gifts from Prof. Noboru Mizushima (The University of Tokyo) (32). The Atg13 KO N2A cells were generated using the CRISPR/Cas9 system. The sgRNA against $\operatorname{Atg} 13$ (5'-GTAGGGAGATTCTATGGAGT-3') was cloned into pSpCas9(BB)-2A-Puro (PX459) V2.0 plasmid (Addgene, 62988), which was transfected into N2A cells using PEI. The cells were then cloned by limiting dilution and cultured. The resultant KO cells were analyzed by immunoblotting and sequencing. Sidt $2^{-/-}$and Sidt $2^{+/+}$MEFs were generated using mouse embryos of the respective genotype on embryonic days 13.5-14.5, and immortalized by transfection of SV40 large T antigen-expressing vector (pBSSVD2005, Addgene, 21826). All cell lines were grown in Dulbecco's modified Eagle's medium (DMEM, Gibco, C11995500) supplemented with 10\% fetal bovine serum.

Antibodies. The following primary antibodies were used in this study: mouse monoclonal anti-DYKDDDDK tag antibody (1E6, Wako, 012-22384), mouse monoclonal anti- $\beta$-actin antibody (AC-15, Sigma-Aldrich, A1978), rabbit monoclonal anti-Atg5 antibody (EPR1755(2), Abcam, ab108327), rabbit polyclonal anti-Atg13 antibody (Sigma-Aldrich, SAB4200100), rabbit monoclonal anti-LC3B antibody (D11, Cell Signaling Technology, 3868S), goat polyclonal anti-GST antibody (GE Healthcare, 27457701), rabbit polyclonal anti- $\alpha$-synuclein antibody (Sigma-Aldrich, AB5038P), mouse monoclonal anti-Tau antibody (Tau46, Cell Signaling Technology, 4019S), rabbit polyclonal anti-LAMP2A antibody (abcam, ab18528), mouse monoclonal anti-VPS4A antibody (VPS4-110, Sigma-Aldrich, SAB4200215), rabbit monoclonal anti-LIMPII antibody (EPR12080, abcam, ab176317), and rabbit polyclonal anti-SIDT2 antibody raised against the N-terminus of human SIDT2 (Abnova, PAB27211). The production of rabbit 
polyclonal antibody against the N-terminus of mouse SIDT2 (NVSQKDAEFERTYA) was outsourced to Eurofins. Rabbit polyclonal antibody against the C-terminal region of human and mouse SIDT2 (DLDTVQRDKIYVF) was produced as previously described (18). membranes (Bio-Rad, 1620177; or Wako, 033-23433), which were then blocked with 3\% bovine serum albumin prepared in DPBS or 5\% skim milk prepared in PBS containing $0.1 \%$ Tween 20. This was followed by incubation with primary antibodies prepared in either of the blocking buffers overnight at $4{ }^{\circ} \mathrm{C}$. The membranes were then washed with $0.1 \%$ Tween 20 prepared in PBS and probed with horseradish peroxidase-conjugated secondary antibodies (Pierce, 31430 or 31460). Signals were then visualized using ImmunoStar Zeta (Wako, 295-72404) or ImmunoStar LD (Wako, 290-69904), and detected using the FluorChem chemiluminescence imaging system (Alpha Innotech) or FUSION chemiluminescence imaging system (Vilber-Lourmat). The signal intensity was quantified using FluorChem or FUSION software.

Measurement of degradation levels of radiolabeled total proteins in cultured cells. A total of $3.0 \times$ $10^{4}$ cells/well of $A t g 5^{+/+}$MEFs or $2.0 \times 10^{4}$ cells/well of $A t g 5^{-/}$MEFs were seeded in 24-well plates (FALCON, 353047). The following day, $37 \mathrm{kBq} /$ well leucine, L-[4,5- $\left.{ }^{3} \mathrm{H}(\mathrm{N})\right]-$

(PerkinElmer, NET135H) was added to the medium and the cells were radiolabeled for $48 \mathrm{~h}$. After $48 \mathrm{~h}$ of labelling, the cells were washed with $500 \mu \mathrm{L} /$ well of medium containing $8 \mathrm{mM}$ unlabeled leucine (Sigma-Aldrich) and incubated in another $500 \mu \mathrm{L} /$ well containing the same medium for 24 h. Following this incubation, the cells were harvested using $500 \mu \mathrm{L} /$ well of trypsin (Gibco, 25200-056) and mixed with the same volume of $10 \%$ trichloroacetic acid (TCA, Wako, 
207-04955). The samples were then chilled on ice, centrifuged at $6,500 \times \mathrm{g}$ and $4^{\circ} \mathrm{C}$ for $10 \mathrm{~min}$, and the TCA-insoluble fraction was collected. The same procedures were also performed after the labelling $(0 \mathrm{~h})$, and TCA-insoluble radioactivity for all samples was measured using Tri-Carb 3100TR (Packard). The degradation levels of protein were measured as the relative decline in radioactivity between 0 and $24 \mathrm{~h}$. For the knockdown experiments, 10 pmol/well siRNA was transfected into cells $4 \mathrm{~h}$ prior to radiolabeling using Lipofectamine 3000 (Thermo Fisher Scientific, L3000-015), in accordance with the manufacturer's instructions.

Measurement of proteolysis in cultured cells using Tet-Off system. A total of $1.5 \times 10^{5}$ cells/well of N2A cells, HeLa Tet-Off Advanced cells (Clontech, 631156) or Atg13 KO N2A cells were seeded in 12-well plates (FALCON, 353043). The cells were transfected with plasmids using polyethyleneimine (PEI, Polysciences, 24765-2) the following day, and treated with $1 \mu \mathrm{g} / \mathrm{mL}$ doxycycline (Clontech, 631311) $24 \mathrm{~h}$ later to stop the expression of the FLAG-tagged proteins. The cells were then incubated for $8 \mathrm{~h}$ to exclude the effect of mRNA degradation and the samples defined as " $0 \mathrm{~h}$ " in the experiment were collected. The cells for samples at other time points were further incubated and collected at each of the indicated time points. The relative levels of FLAG-tagged proteins were quantified using immunoblotting and the degradation levels were calculated by subtracting the relative levels of proteins at each time point (percent in relation to the $0 \mathrm{~h}$ time-point) from $100 \%$. N2A cells were transfected with $0.16 \mu \mathrm{g} /$ well of pTet-Off vector, 0.02 $\mu \mathrm{g} /$ well of pTRE-Tight vector expressing each FLAG-tagged protein, and pCI-neo vector expressing EGFP, WT, 3 YS, 3RS, or S564A mouse SIDT2. For experiments using inhibitors, 50 $\mu \mathrm{g} / \mathrm{mL}$ cycloheximide (Sigma-Aldrich, C1988-1G) or $50 \mu \mathrm{g} / \mathrm{mL}$ pepstatin A (Peptide Institute, 4397-v), and $50 \mu \mathrm{g} / \mathrm{mL}$ E64d (Peptide Institute, 4321-v) were added to the medium at the time 
point " 0 h". For the experiment shown in Fig. 4G, N2A cells were transfected with $0.10 \mu \mathrm{g} / \mathrm{well}$ of pTet-Off vector, $0.02 \mu \mathrm{g} /$ well of pTRE-Tight- $\alpha$-synuclein-FLAG, and $0.08 \mu \mathrm{g} /$ well of pCI-neo vector expressing EGFP or human $\triangle 2226 \mathrm{G}$ SIDT2. For the experiment shown in Fig. 4K, N2A cells were transfected with $0.10 \mu \mathrm{g} /$ well of pTet-Off vector, $0.02 \mu \mathrm{g} /$ well of pTRE-Tight- $\alpha$-synuclein-FLAG, $0.02 \mu \mathrm{g} /$ well of pCI-neo-WT human SIDT2, and $0.08 \mu \mathrm{g} /$ well of pCI-neo vector expressing EGFP or human $\triangle 2226$ G SIDT2. For the knockdown experiments, N2A cells were transfected with $0.10 \mu \mathrm{g} /$ well of pTet-Off vector, $0.05 \mu \mathrm{g} /$ well of pTRE-Tight- $\alpha$-synuclein-FLAG, $0.05 \mu \mathrm{g} /$ well of pCI-neo vector expressing EGFP or mouse SIDT2, and $10 \mathrm{pmol} /$ well of siRNA using Lipofectamine 3000, in accordance with the manufacturer's instructions. HeLa Tet-Off Advanced cells were transfected with $0.10 \mu \mathrm{g} / \mathrm{well}$ of pTRE-Tight- $\alpha$-synuclein-FLAG and $0.10 \mu \mathrm{g} /$ well of pCI-neo vector expressing EGFP or mouse SIDT2.

Preparation of recombinant GST and GST-tagged proteins. The GST and GST-tagged proteins were prepared as previously described (19), with slight modifications. Briefly, protein expression was induced in Rosetta2 (DE3) competent cells (Millipore, 71397) using the pGEX-6P-1 vector and $500 \mu \mathrm{M}$ isopropyl- $\beta$-D(-)-thiogalactopyranoside (IPTG, Wako, 094-05144) at $25^{\circ} \mathrm{C}$ for $17 \mathrm{~h}$ and harvested by centrifugation. The cells were then resuspended in PBS containing $1 \%$ Triton-X and protease inhibitors (Roche) and lysed by sonication. The sample was then centrifuged at $4^{\circ} \mathrm{C}$ for $10 \mathrm{~min}$ at $18,000 \times \mathrm{g}$, and the proteins were purified from the supernatant using Glutathione Sepharose 4B (GE Healthcare, 17-0756-01). Purified proteins were then incubated on ice for 10 min in $1 \mathrm{M} \mathrm{NaCl}$, and then the buffer was dialyzed against PBS using Slide-A-Lyzer Dialysis Cassette, 10,000 MWCO, 0.5 mL (Thermo Fisher Scientific). 
Pull-down assay. Glutathione Sepharose 4B was blocked with 3\% bovine serum albumin in PBS overnight at $4^{\circ} \mathrm{C}$ and washed three times with PBS containing $1 \%$ Triton-X 100. Next, $15 \mu \mathrm{g}$ of recombinant His-tagged $\alpha$-synuclein was incubated with 8 nM GST or GST-tagged proteins and the blocked beads for $18 \mathrm{~h}$ at $4^{\circ} \mathrm{C}$ in PBS containing $1 \%$ Triton-X 100. Following this incubation, the beads were washed with the same buffer three times and the pulled-down protein was extracted using SDS sample buffer (10 mM Tris, pH 7.8, 3\% SDS, 5\% glycerol, 0.02\% bromophenol blue, and 2\% 2-mercaptoethanol). were seeded in $35 \mathrm{~mm}$ glass-bottomed dishes (IWAKI, 3970-035), transfected with $0.32 \mu \mathrm{g} / \mathrm{dish}$ of pTet-Off vector, $0.04 \mu \mathrm{g} /$ dish of pTRE-Tight- $\alpha$-synuclein-mCherry, and $0.04 \mu \mathrm{g} /$ well of pCI-neo (empty vector) or pCI-neo-mouse Sidt2, and then added with $1 \mu \mathrm{g} / \mathrm{mL}$ doxycycline one day later. The following day, the cells were treated with LysoTracker Green DND-26 (Life Technologies, L7526) to label the lysosomes and then analyzed using an FV10 confocal microscope (Olympus). Quantification and calculation of colocalization rates were performed with ImageJ software via the JACoP plugin. For the experiment shown in Fig. S10C, $3.0 \times 10^{5}$ cells/dish of N2A cells were seeded in $35 \mathrm{~mm}$ glass-bottomed dishes, and the cells were transfected with $0.16 \mu \mathrm{g} / \mathrm{well}$ of pmCherry-N1-human $\triangle 2226 \mathrm{G}$ SIDT2 with $0.24 \mu \mathrm{g} /$ well of pCI-neo (empty vector). The following day, the cells were treated with LysoTracker Green DND-26 to label the lysosomes and then analyzed using an FV10 confocal microscope. For the experiment shown in Fig. S7A, $3.0 \times 10^{5}$ cells/dish of N2A cells were seeded in $35 \mathrm{~mm}$ glass-bottomed dishes. The following day, the cells were transfected with $0.28 \mu \mathrm{g} / \mathrm{dish}$ of pTet-Off vector, $0.04 \mu \mathrm{g} / \mathrm{dish}$ of 
pTRE-Tight- $\alpha$-synuclein-mCherry, $0.04 \mu \mathrm{g} /$ well of pEGFP-N1-Lamp1, and $0.04 \mu \mathrm{g} /$ well of pCI-neo-mouse Sidt2, and then treated with $1 \mu \mathrm{g} / \mathrm{mL}$ doxycycline one day later. The following day, the fluorescence in single cells was analyzed using DeltaVision Elite (Applied Precision). For the experiment shown in Fig. S7B, $3.0 \times 10^{5}$ cells/dish of N2A cells were seeded in $35 \mathrm{~mm}$ glass-bottomed dishes. The following day, the cells were transfected with $0.32 \mu \mathrm{g} / \mathrm{dish}$ of pTet-Off vector, $0.04 \mu \mathrm{g} / \mathrm{dish}$ of pTRE-Tight- $\alpha$-synuclein-Egfp-mCherry, $0.04 \mu \mathrm{g} /$ well of pCI-neo (empty vector), or pCI-neo-mouse Sidt2, and then treated with $1 \mu \mathrm{g} / \mathrm{mL}$ doxycycline one day later to stop the expression of $\alpha$-synuclein-EGFP-mCherry. The next day, the cells were treated with LysoTracker BlueDND-22 (Life Technologies, L7525) to label the lysosomes and then analyzed using an FV10 confocal microscope.

Measurement of autophagic flux. For the measurement of the autophagic flux at steady-state levels shown in Fig. S8A, $1.5 \times 10^{5}$ cells/well of WT N2A cells were seeded in 12-well plates. The following day, the cells were transfected (using PEI) with $0.16 \mu \mathrm{g} /$ well of pCI-neo (empty vector), $0.02 \mu \mathrm{g} /$ well of pCI-neo (empty vector), or pCI-neo-mouse Sidt2, $0.02 \mu \mathrm{g} /$ well of pMRX-IP-GFP-RFP-LC3 $\triangle \mathrm{G}(33)$, and then the fluorescence was measured two days later using a SpectraMax i3x (Molecular Devices). Autophagic flux was measured as the ratio of fluorescence between GFP and RFP in each well. For the experiment to analyze whether the cells are capable of driving autophagy (34) in Fig. S8E, the protocol was as follows. Briefly, $1.5 \times 10^{5}$ cells/well of WT or Atg13 KO N2A cells were seeded in 12-well plates, and the medium was replaced with amino acid starvation medium (Wako, 048-33575) with or without $100 \mathrm{nM}$ bafilomycin A1, or normal medium as a control, for $2 \mathrm{~h}$. The samples were then subjected to immunoblotting against 
LC3, and the autophagic flux was analyzed as the difference in the relative levels of LC3-II and LC3-I.

$\underline{\text { In vitro reconstitution of direct uptake and degradation of protein by isolated lysosomes. }}$

Experiments using isolated lysosomes were performed as previously described $(3,4,7,8,18,19$,

35) with slight modifications. Briefly, lysosomes were isolated from MEFs, N2A cells, mouse

brain, or skeletal muscles using the Lysosome Enrichment Kit for Tissue and Cultured Cells

(Thermo Scientific, 89839) and suspended in $300 \mathrm{mM}$ sucrose. Lysosomes were then mixed with

$0.1 \mu \mathrm{g} / \mathrm{assay}$ of recombinant $\alpha$-synuclein (BIOMOL, SE-256) or tau (tau-441, Wako, 205-20331)

protein and 10 mM 3-(N-morpholino)propanesulphonic acid (MOPS, Dojin, 345-01804) buffer

(pH 7) with or without $10 \mathrm{mM}$ (final concentration) adenosine 5'-triphosphate disodium salt

n-hydrate (ATP-Na, Wako 017-09673) in $300 \mathrm{mM}$ sucrose, and incubated at $37^{\circ} \mathrm{C}$ for $0 \mathrm{~min}, 5$

min, $10 \mathrm{~min}$, or $20 \mathrm{~min}$. The samples were then subjected to immunoblotting and the relative levels

of $\alpha$-synuclein or tau were quantified. The degradation of $\alpha$-synuclein was obtained by subtracting

15 the relative levels of $\alpha$-synuclein (percent of input) from $100 \%$. Of note, for the experiment shown in Fig. S2C, the samples were centrifuged following incubation $\left(4^{\circ} \mathrm{C}, 17,700 \times \mathrm{g}, 1 \mathrm{~min}\right)$, and the relative levels of proteins in the resultant supernatants and the lysosomal pellets were analyzed by immunoblotting. For the experiment shown in Fig. 1D, the suspended lysosomes were preincubated with or without $50 \mu \mathrm{g} / \mathrm{mL}$ pepstatin A and $50 \mu \mathrm{g} / \mathrm{mL}$ E64d for $30 \mathrm{~min}$ on ice, and then subjected to the following experiments. For the experiments shown in Fig. 1G and Fig. S2E, the suspended lysosomes were preincubated with or without $1.0 \mu \mathrm{g} / \mathrm{assay}$ of recombinant His-tagged Hsc70 protein for $30 \mathrm{~min}$ on ice, and then subjected to the following experiments. For the experiment shown in Fig. S2B, isolated lysosomes were incubated at $37^{\circ} \mathrm{C}$ for $20 \mathrm{~min}$ in the 
presence or absence of ATP and precipitated by centrifugation $\left(4^{\circ} \mathrm{C}, 17,700 \times \mathrm{g}, 1 \mathrm{~min}\right)$. The supernatant was collected and then incubated with $0.1 \mu \mathrm{g} /$ assay of recombinant $\alpha$-synuclein protein at $37^{\circ} \mathrm{C}$ for $20 \mathrm{~min}$. For the experiment shown in Fig. S4B, the isolated lysosomes suspended in $300 \mathrm{mM}$ sucrose were dissolved in citrate buffer containing 1\% Triton-X (final volume), and then incubated with $0.1 \mu \mathrm{g} /$ assay of recombinant $\alpha$-synuclein at $37^{\circ} \mathrm{C}$ for $5 \mathrm{~min}$. For the experiments shown in Fig. 5I and Fig. S11F, lysosomes were isolated from skeletal muscles of the lower extremities of mice and subjected to the following experiments. For the degradation of RNA, $5.0 \mu \mathrm{g} / \mathrm{assay}$ of total RNA was used. In the experiments using lysosomes derived from distinct samples, the lysosome amount was confirmed by immunoblotting on LIMPII.

Measurement of lysosomal pH. A total of $2.0 \times 10^{5}$ cells/well of Sidt2 ${ }^{+/+}$and Sidt2 ${ }^{-/-}$MEFs were seeded in $35 \mathrm{~mm}$ glass-bottomed dishes, and cells were treated with or without $100 \mathrm{nM}$ bafilomycin A1, followed by 15 min of treatment with $1 \mu \mathrm{M}$ LysoSensor Green DND-189 (Thermo Fisher Scientific, L7535) $1 \mathrm{~h}$ later. The medium was then washed out, cells were incubated for $1 \mathrm{~h}$ and lysosomal $\mathrm{pH}$ was analyzed as relative fluorescence units using an FV10 confocal microscope and ImageJ software.

Immunoelectron microscopy. Immunoelectron microscopy was performed as previously described $(3,4,7,8)$. Briefly, isolated lysosomes were incubated in the presence of ATP with or without recombinant $\alpha$-synuclein protein and $5 \mathrm{~nm}$ gold particles (Cytodiagnostics, G-5-20), and then precipitated by centrifugation. Lysosomes were then fixed with $0.1 \%$ glutaraldehyde and $4 \%$ paraformaldehyde in $0.1 \mathrm{M}$ phosphate buffer overnight at $4^{\circ} \mathrm{C}$, dehydrated in a series of water/ethanol mixtures to $100 \%$ ethanol, and subsequently embedded in LR White (Nisshin EM, 
3962). The samples were then sectioned at a thickness of $100 \mathrm{~nm}$ and collected on 400-mesh nickel grids. The sections were subsequently immunolabelled with anti- $\alpha$-synuclein antibody (Cell Signaling Technology, 2628 for Fig. 1E and Fig. S3B; and Abcam, ab138501 for Fig. S3A and C), followed by $15 \mathrm{~nm}$ gold-labelled goat anti-rabbit $\operatorname{IgG}(\mathrm{H}+\mathrm{L})$ (Amersham), and observed using a Tecnai Spirit transmission electron microscope (FEI) at $80 \mathrm{kV}$.

Preparation of recombinant $\triangle \mathrm{DQ}$ mutant $\alpha$-synuclein protein. The $\triangle \mathrm{DQ} \alpha$-synuclein recombinant protein was prepared as previously described (3), with slight modifications. Briefly, Rosetta2 (DE3) competent cells (Millipore, 71397) were transformed with pGEX-6P-1- $\triangle \mathrm{DQ} \alpha$-synuclein and the protein expression was induced with $500 \mu \mathrm{M}$ isopropyl- $\beta$-D(-)-thiogalactopyranoside (IPTG, Wako, 094-05144). The cells were incubated at $25^{\circ} \mathrm{C}$ overnight and harvested by centrifugation. The cells were then suspended in PBS containing 1\% Triton-X and protease inhibitors and lysed by sonication. The sample was then centrifuged for $10 \mathrm{~min}$ at $18,000 \times g$ and $4^{\circ} \mathrm{C}$, and GST-tagged $\triangle \mathrm{DQ} \alpha$-synuclein was purified from the supernatant using Glutathione Sepharose 4B (GE Healthcare, 17-0756-01). $\triangle \mathrm{DQ} \alpha$-synuclein protein was then cleaved from GST using PreScission Protease (GE Healthcare, 27-0843-01).

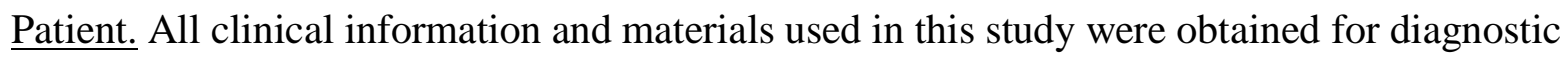
purposes with written informed consent. The ethics committee of the National Center of Neurology and Psychiatry (NCNP) approved this study and the use of human subjects for this study. All relevant ethical regulations were followed. We reviewed the medical information obtained from interviews conducted by physicians and evaluated the patient's clinical manifestations, disease course, and laboratory data. 
Skeletal muscle imaging. Skeletal muscle MRI data in digital imaging and communications in medicine format were available as part of the clinical information at the time of muscle pathology diagnosis at the NCNP.

Mutation analysis. At the NCNP, whole-exome sequencing (WES) analysis has been performed in 2,131 undiagnosed cases of suspected hereditary muscle disease using genomic DNA from blood or biopsied muscle samples (Neurology 2020, in press). We screened this WES database for the variants in SIDT2 and evaluated them according to the following variables: (1) mutation effect (i.e., splicing, start lost, stop gained or lost, frameshift, nonsynonymous codon change, and codon insertion or deletion); and (2) variation frequency, $<0.001$ in a public database [Genome Aggregation Database (gnomAD), 1000 Genomes Project, or Exome Sequencing Project v. 6500 (ESP6500)], and <0.01 for in-house data (36). The identified variants were confirmed by Sanger sequencing. Expression of the transcript with variants was analyzed by direct sequencing and cloning of the RT-PCR products of the SIDT2 transcript from skeletal muscles of patients. Primer sequences for the PCR are available upon request.

Muscle pathology. Muscle samples were collected from the deltoid muscle of the patient and from the quadriceps femoris, gastrocnemius, and tibialis anterior muscles of mice and then frozen in isopentane cooled in liquid nitrogen (37). Histological analysis was performed as previously described (38). Electron microscopy-based observation of the hamstring muscles from the mice was performed in line with a previously reported protocol (39). Immunofluorescence of the 
skeletal muscles was performed in accordance with previously reported methods (25). For the morphometric analysis, we stained frozen transverse sections of gastrocnemius muscles for caveolin 3, LC3, and p62 and acquired images under a Keyence BZ X-700 microscope. Five randomly selected images (per mouse) with caveolin 3 staining were used to measure fiber diameters using ImageJ (NIH). The minimal inner diameters of 2,000 myofibers from each mouse were measured. Images of whole muscles were used to measure RVs and inclusion by LC3 and p62 staining, respectively (40). The following primary antibodies were used in this study: anti-LAMP2 mouse-Mono (H4B4, Santa Cruz), anti-p62 Rb-poly (BML-PW9860, Enzo), anti-LC3 Rb-poly (NB100-2220, Novus), anti-Cav3 goat-poly (N-18 Santacruz), anti-amyloid mouse-Mono (6E-10, Covance), and anti-TDP-43 Rb-poly (Protein Tech).

In vitro de-glycosylation of SIDT2 using PNGase F. N2A cells expressing GFP, WT human SIDT2, $\triangle 2226 \mathrm{G}$ human SIDT2, or WT and $\Delta 2226 \mathrm{G}$ human SIDT2 were lysed with $1 \%$ Triton X-100 lysis buffer (50 mM Tris-HCl, pH 7.5, 150 mM NaCl, 5 mM EDTA, $1 \%$ Triton X-100) 15 containing protease inhibitors (Complete, EDTA free; Roche Applied Science, 1873580), and treated with PNGase F (New England BioLabs, P0704S) at $37^{\circ} \mathrm{C}$ for 4 h or left untreated. Following the treatment, the samples were subjected to immunoblotting.

Generation of Sidt2 knockout mice. Sidt $2^{-/-}$mice were generated using the CRISPR/Cas9 system. The sequences for sgRNA against two distinct sites in exon 13 (5'-ACTGGACTCCATGAGCTCCG-3') and exon 26 (5'-CGACTTGGACACAGTACAGC-3') of SIDT2 were designed using the CRISPR DESIGN program (http://crispr.mit.edu) and cloned into the pX330 plasmid (Addgene, 42230). The plasmids were then directly injected into pronuclei 
of B6C3F1 fertilized eggs. The genotype of the resultant KO mice (deletion between exon 13 and 26) was confirmed by PCR with following primers:

5'-GGGACCTCTCCTACAGTTACCAGG-3', 5'-CCGCAGAGGCAACTCCAAGGCTC-3', and 5'-CGCAGAGTCCAGGGAGCACAAC-3'. The KO mice were backcrossed with C57BL/6J mice

for at least eight generations before being used in the experiments; the data shown in Fig. 5, D to H and Fig. S11, F, G, and I are exceptions as in these cases, backcrossing was done for three generations. We confirmed that rimmed vacuoles were also observed in the backcrossed mice (eight generations). All animal experiments were approved by the animal committee of the NCNP and all relevant ethical regulations were followed. Mice were maintained under specific

10 pathogen-free conditions.

Physiological examination of mice. Grip strength of combined forelimbs/hindlimbs and voluntary locomotion within an individual cage using a running wheel were measured as described previously (38). The contractile properties of the tibialis anterior and gastrocnemius muscles were measured in accordance with previously reported protocols $(41,42)$.

Quantification of relative transcript expression in $\operatorname{Sid}_{\mathrm{t}}{ }^{+/+}$and Sidt $2^{-/-}$mouse muscles. RNA was purified from frozen sections of mouse gastrocnemius using the TRI Reagent (Sigma-Aldrich, T9424), in accordance with the manufacturer's instructions. cDNA was then synthesized using PrimeScript RT Reagent Kit with gDNA Eraser (Perfect Real Time) (TaKaRa, RR047) and employed as a template for quantitative PCR on the CFX96 Touch Real-Time PCR Detection System (Bio-Rad). TB Green Premix Ex Taq II (Tli RNaseH Plus) (TaKaRa, RR820) was used for performing the quantitative PCR. The levels of each target RNA were normalized to those of 
Gapdh. The following primers were used for the detection of respective RNAs: 28S rRNA

Forward: 5'-TGGGAATGCAGCCCAAAGC-3', Reverse:

5'-CCTTACGGTACTTGTTGACTATVG-3'; 18S rRNA Forward:

5'-GTAACCCGTTGAACCCCATT-3', Reverse: 5'-CCATCCAATCGGTAGTAGCG-3'; App

Forward: 5'-GGAAGCAGCCAATGAGAGAC-3', Reverse:

5'-GGTTCATGCGCTCGTAGATCA-3'; p62 Forward:

5'-TGTGGAACATGGAGGGAAGAG-3', Reverse: 5'-GTGCCTGTGCTGGAACTTTC-3';

$\alpha$-synuclein Forward: 5'-ATGGATGTGTTCATGAAAGGAC-3', Reverse:

5'-AACCACTCCTTCCTTAGTTTTG-3'; Tau Forward: 5'-

CCTGAGCAAAGTGACCTCCAAG-3', Reverse: 5'-CAAGGAGCCAATCTTCGACTGG-3';

Tdp43 Forward: 5'-CTCAGTGTATGAGAGGAGTCCGAC-3', Reverse:

5'-ACACTATGAGGTCAGATGTTTTCTGG-3'; and Lc3 Forward: 5'-

GACGGCTTCCTGTACATGGTTT-3', Reverse: 5'-TGGAGTCTTACACAGCCATTGC-3'.

Specific primers for 5S rRNA, 5.8S rRNA, and Gapdh were prepared as previously described (8).

Atg7 skeletal muscle conditional knockout mice. Atg7-flox mice were developed by Prof Komatsu Masaaki (Juntendo University) (43) and MuCre-A mice were developed by Prof Kyoko Koishi (University of Otago) (44) (20877696). Both mouse models were provided by the Riken BioResource Research Center (RBRC02759 and RBRC01386). Mice with skeletal muscle-specific Atg7 KO were generated by mating. 
Preparation of feeding and fasting mice. For starvation, mice were placed in clean cages without food for $30 \mathrm{~h}$ (from 9 AM to 3 PM the next day). Water was provided ad libitum. Starvation for 30 h resulted in a $10 \%$ loss of weight (starvation did not result in mortality). two groups and by Dunnett's or Tukey's multiple comparison test for more than two groups. All statistical tests were two-sided. All measurements were taken from distinct samples. For immunoblotting and for microscopy-based images with quantitative data, representative images are shown. Graphs show means, data points, and SE. 


\section{Supplementary Text}

\section{Supplementary text A}

The best characterized autophagic pathway is macroautophagy, where various cellular components are sequestered by autophagosomes, double-membraned vesicles, and delivered to lysosomes. In chaperone-mediated autophagy (CMA), chaperones assist the direct import of specific groups of proteins into lysosomes, while in microautophagy, cytosolic components are sequestered into lysosomes/endosomes by invagination. In mammals, the mechanism underlying microautophagy has only been described in terms of the endosomal pathway $(15,16)$.

\section{Supplementary text B}

The contributions of the non-macroautophagic pathways to the degradation of proteins were investigated under steady-state conditions (a nutrient-rich condition). Macroautophagy is primarily an inducible pathway that responds to stresses such as starvation (1).

\section{Supplementary text C}

In autophagic pathways other than macroautophagy, lysosomes are directly involved in the internalization of substrates. Therefore, our observation of macroautophagy-independent lysosomal proteolysis led us to hypothesize that direct uptake and degradation of protein may be occurring in lysosomes. 


\section{$\underline{\text { Supplementary text D }}$}

To investigate the ATP-dependent steps in the above mechanism, we isolated the lysosomes following the incubations, and determined the relative amounts of substrate protein localized in the lysosome fraction (including those interacting with the lysosomal membrane) and the assay solution. Although some of the substrate proteins partially co-localized with lysosomes even in the absence of ATP, protein degradation was only observed in the presence of ATP (Fig. S2C).

\section{Supplementary text E}

Among the known subtypes of autophagy in mammals, the only pathways reconstituted in isolated lysosomes that directly take up macromolecules for subsequently degradation are CMA and RDA. Thus, we investigated whether this novel pathway is related to these known pathways.

\section{Supplementary text F}

Direct uptake of protein by CMA has been reported to occur in a specific sub-group of lysosomes in the absence of the metrizamide reagent, which we did not use in our experiments (46).

Altogether, based on the results of our experiments, we predict that CMA-active/competent lysosomes are not enriched in our experimental condition. 


\section{$\underline{\text { Supplementary text G }}$}

The protein products of Lamp2a and Vps4 are required for CMA (14) and microautophagy in late endosomes $(15,16)$, respectively.

\section{Supplementary text $\mathrm{H}$}

Among the various proteins examined, the contribution of SIDT2 to the degradation of tau and $\alpha$-synuclein protein was clearly indicated, as the protein was markedly degraded under SIDT2 overexpression, while no significant degradation of these proteins was observed in control cells (Fig. 1, C and D).

\section{Supplementary text I}

First, we demonstrated that SIDT2-dependent proteolysis and macroautophagy are independent processes. Thus, the basal macroautophagy was not increased upon SIDT2 overexpression (Fig. S8A), and the SIDT2-dependent increase in proteolysis was independent of Atg5, and Atg13, a macroautophagy-initiation factor (Fig. S8, B to G). In addition, SIDT2 did not significantly increase the degradation of LC3 protein (Fig. S8H). Next, we confirmed that SIDT2-dependent lysosomal proteolysis and CMA are independent processes. Using the Tet-Off system, we observed that $\triangle \mathrm{DQ}$ mutant $\alpha$-synuclein $(10)$ and $\triangle \mathrm{NR}$ mutant GAPDH proteins, both of which lack KFERQ-like motifs, are still degraded in an SIDT2-dependent manner at the cellular level proteolysis in cells (Fig. 2I). Because the overexpression of LAMP2A did not affect the degradation rate of $\alpha$-synuclein, it is likely that CMA was not active under our experimental 
conditions (Fig. S9, A and B). Thus, SIDT2 mediates the lysosomal degradation of proteins independent of CMA. Finally, we confirmed that SIDT2-dependent lysosomal proteolysis and microautophagy are independent processes. In mammalian cells, microautophagy in the late endosomes is also partly dependent on hsc70 and the KFERQ-like motif (15). In addition, microautophagy has been demonstrated to require Atg13 in Drosophila (47). SIDT2-dependent proteolysis occurs in the presence of the Vps4 knockdown (Fig. 2J), providing definitive evidence that this pathway is also independent of endosomal microautophagy. Together, these observations indicate that SIDT2-dependent proteolysis is a novel pathway for autophagy.

Basically, all known receptors for microautophagy and macroautophagy—as well as membrane-bound LC3 in macroautophagy_are degraded along with their substrate as a consequence of their interaction. However, considering that the binding of SIDT2 to the substrate is required for this pathway (Fig. 1, H and I) and as a decline in the level of SIDT2 was not observed during the process (Fig. 2G), SIDT2 is unlikely to function in a mechanism akin to these known pathways, or through microautophagy via an as-yet-unknown mechanism. These facts suggest that SIDT2 functions as a transporter-rather than a mere receptor-in this pathway.

\section{Supplementary text K}

Defects in intracellular degradation systems that are fundamental to homeostasis often lead to the development of diseases characterized by an accumulation of intracellular deposits, typically in the neurons and muscles. 


\section{Supplementary text L}

The symptoms began in the third decade of life with gradual progression of muscle weakness and atrophy in the upper and lower extremities. The patient developed an inability to run at the age of 54 years owing to a bilateral foot drop, and an inability to perform fine handiworks at 59 years of age. The patient's father and son were also affected (Fig. 3A). Electromyography and nerve conduction revealed early recruitment and markedly lower amplitudes of motor unit action potential and compound muscle action potential.

Upon radiography of the patient, hamstring, triceps surae, and tibialis anterior muscles were preferentially replaced by fat (Fig. 3B). His vastus lateralis muscles showed marked variation in myofiber size, small group atrophy with several small angulated fibers and pyknotic nuclear clumps, fibrosis, and adipose tissue infiltration in the endomysium (Fig. 3C). Rimmed vacuoles, in which aggregate-prone proteins were characteristically accumulated along with autophagic and lysosomal proteins, were observed in some atrophic myofibers (Fig. 3, D and E). In addition to those myogenic observations, a remarkable deficit in axons of the intramuscular nerves was also observed (Fig. 3F).

$\operatorname{Sidt} 2^{-/}$mice exhibited a smaller size, a reduction in locomotor activity, and decreased grip strength (Fig. S9, B to D). A progressive and severe decrease in muscle contraction and a gradual 
reduction in the size of skeletal muscles, characteristic of the myopathic phenotypes, were also observed (Fig. 4, A to D and Fig. S9E). Histological analysis revealed that the myofibers were atrophic and varied in size, while no necrotic and regenerating fibers were observed (Fig. 4E). In addition, fibrosis and adipocyte infiltration were also observed (Fig. 4E), all of which were

strikingly analogous to those seen in the muscle of the patient. Indeed, rimmed vacuoles immunoreactive to various aggregate-prone proteins and LC3 were observed in the skeletal myofibers of Sidt $2^{-/-}$mice (Fig. 4F). Biochemically, accumulation of $\alpha$-synuclein was also observed in the skeletal muscle of these mice (Fig. 4G) Ultrastructurally, amorphous inclusions surrounded by multilamellar bodies (25) at the center of myofibers in Sidt $2^{-1-}$ mice were observed (Fig. 4H). Taken together, these features clearly show that SIDT2-deficient mice recapitulate the typical symptoms of rimmed vacuolar myopathy.

\section{$\underline{\text { Supplementary text } \mathrm{O}}$}

In general, all RVMs are thought to be primarily caused by the cytoplasmic accumulation of proteins and other substances that should constitutively be degraded; aberrancy in the normal functions of lysosomes such as luminal $\mathrm{pH}$ and hydrolase activity is not the primary cause of this group of myopathies (23). This is consistent with the fact that all causative genes of RVMs reported previously encode extra-lysosomal proteins. To our knowledge, this is the first causative gene of RVM that encodes a lysosomal protein. This is in contrast to the fact that all known causative genes for autophagic vacuolar myopathies (AVMs) encode lysosomal proteins, and these diseases are thought to be primarily caused as a result of lysosomal aberrations (23). Importantly, none of these primary lysosomal myopathies are characterized by rimmed vacuoles. These findings strongly suggest that SIDT2 deficiency causes RVM through impairment of the 
translocation of degradation substrates from the cytoplasm to the lysosomal lumen by DUMP, rather than through mere impairment in the normal function of lysosomes in the lumen.

\section{$\underline{\text { Supplementary text } \mathrm{P}}$}

Similar to the observations in the patient, the accumulations of LC3, multilamellar bodies, and clusters of autophagosomes were also seen in the cytosolic inclusions in the muscles of SIDT2-deficient mice (Fig. 5, F and H). These features are probably the consequences of the secondary induction of macroautophagy in response to inclusion formation, as commonly seen in other rimmed vacuolar myopathies $(23,48)$. In fact, we observed that macroautophagy was activated upon fasting in the muscles of Sidt $2^{-/-}$mice (Fig. S9H). In addition, histological and immunohistological features of the muscles in skeletal muscle-specific conditional Atg7 KO mice were distinct from those seen in Sidt $2^{-/-}$mice (Fig. S9I), providing further support for the proposal that the myopathic features of Sidt $2^{-/-}$mice and the patient are unlikely to be the consequence of macroautophagy deficiency.

\section{$\underline{\text { Supplementary text Q }}$}

Macroautophagy-deficient muscles did not harbor rimmed vacuoles, exhibiting pathological features distinct from those of SIDT2-deficient muscles (Fig. S11, H and I). This is also the case for the Lamp $2^{-/-}$mouse model, which is predicted to be deficient for CMA, and which exhibits pathway. 


\section{$\underline{\text { Supplementary text R }}$}

Additional cellular mechanisms likely include macroautophagy, the unfolded protein response in the endoplasmic reticulum (ERAD), and the ubiquitin-proteasome system (UPS). Cytosolic degradation pathways such as ERAD and UPS have previously been implicated in the extra-lysosomal cytoplasmic accumulation of proteins in RVMs.

\section{References and Notes:}

32. A. Kuma et al., The role of autophagy during the early neonatal starvation period.

Nature 432, 1032-1036 (2004).

64, 835-849 (2016).

34. T. Kaizuka, N. Mizushima, Atg13 Is Essential for Autophagy and Cardiac Development in Mice. Mol Cell Biol 36, 585-595 (2016).

35. K. Hase et al., RNautophagy/DNautophagy possesses selectivity for RNA/DNA substrates. Nucleic Acids Res 43, 6439-6449 (2015).

36. Y. Endo et al., Dominant mutations in ORAI1 cause tubular aggregate myopathy with hypocalcemia via constitutive activation of store-operated $\mathrm{Ca}(2)(+)$ channels. Hum Mol Genet 24, 637-648 (2015).

37. M. C. Malicdan, S. Noguchi, I. Nishino, Monitoring autophagy in muscle diseases. Methods Enzymol 453, 379-396 (2009).

38. S. Noguchi, M. Ogawa, M. C. Malicdan, I. Nonaka, I. Nishino, Muscle Weakness and Fibrosis Due to Cell Autonomous and Non-cell Autonomous Events in Collagen VI Deficient Congenital Muscular Dystrophy. EBioMedicine 15, 193-202 (2017). 
39. H. T. Nguyen et al., Small-Vessel Vasculopathy Due to Aberrant Autophagy in LAMP-2 Deficiency. Sci Rep 8, 3326 (2018).

40. A. Cho et al., Sialic acid deficiency is associated with oxidative stress leading to muscle atrophy and weakness in GNE myopathy. Hum Mol Genet 26, 3081-3093 (2017). with muscle atrophy and precedes the development of inclusion body or rimmed vacuoles in the mouse model of DMRV/hIBM. Physiol Genomics 35, 106-115 (2008).

42. M. C. Malicdan, S. Noguchi, Y. K. Hayashi, I. Nonaka, I. Nishino, Prophylactic treatment with sialic acid metabolites precludes the development of the myopathic phenotype in the DMRV-hIBM mouse model. Nat Med 15, 690-695 (2009).

43. M. Komatsu et al., Impairment of starvation-induced and constitutive autophagy in Atg7-deficient mice. J Cell Biol 169, 425-434 (2005).

44. M. Azhar et al., Myocardial deletion of Smad4 using a novel alpha skeletal muscle actin Cre recombinase transgenic mouse causes misalignment of the cardiac outflow tract. Int J Biol Sci 6, 546-555 (2010).

45. A. M. Cuervo, J. F. Dice, E. Knecht, A population of rat liver lysosomes responsible for the selective uptake and degradation of cytosolic proteins. J Biol Chem 272, 5606-5615 (1997). 46. S. Kaushik, A. M. Cuervo, Chaperone-mediated autophagy. Methods Mol Biol 445, 227-244 (2008). 


\section{M. C. Malicdan, S. Noguchi, I. Nishino, Autophagy in a mouse model of distal}

myopathy with rimmed vacuoles or hereditary inclusion body myopathy. Autophagy 3, 396-398 (2007). 
A

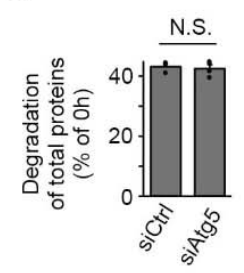

B

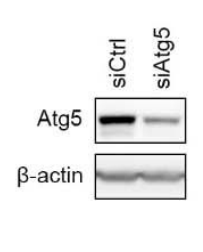

C

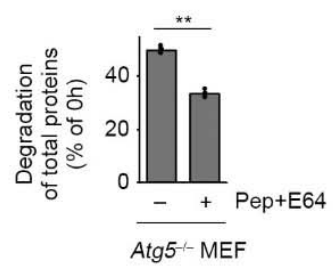

Fig. S1. Contribution of SID2 and canonical macroauthophagy genes to steady-state levels

\section{of proteolysis.}

(A) Effect of Atg5 knockdown on the degradation of radiolabeled total proteins in $\operatorname{Atg} 5^{+/+}$MEFs

$5 \quad(\mathrm{n}=4)$. (B) Expression levels of Atg5 in Atg $5^{+/+}$MEFs knocked down with Atg5. (C) Effect of

lysosomal protease inhibitors on the degradation of radiolabeled total proteins in $\operatorname{Atg} 5^{-/-}$MEFs (n =4). 
A

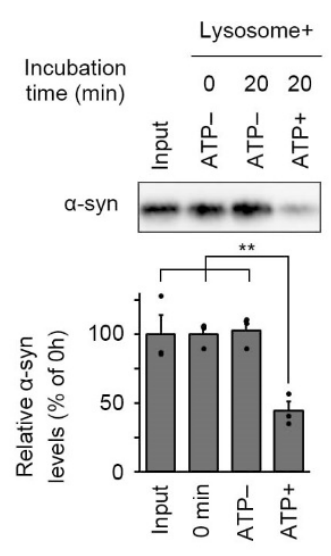

B

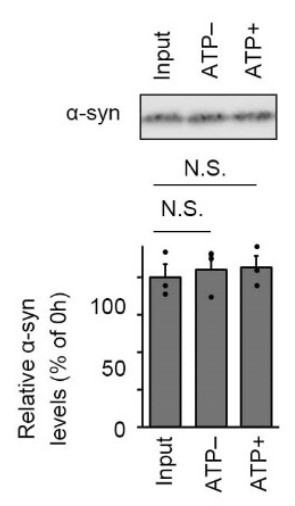

C

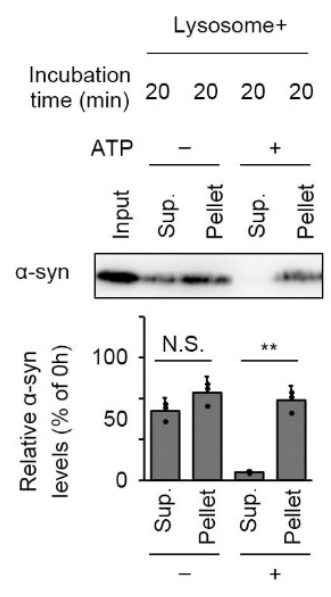

D

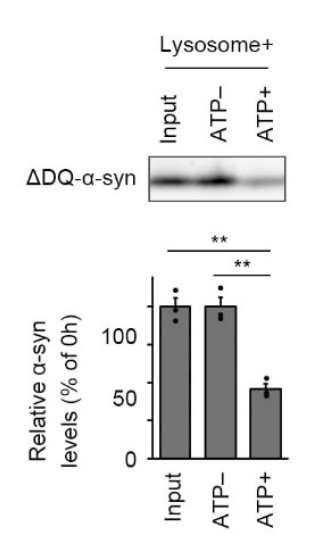

E

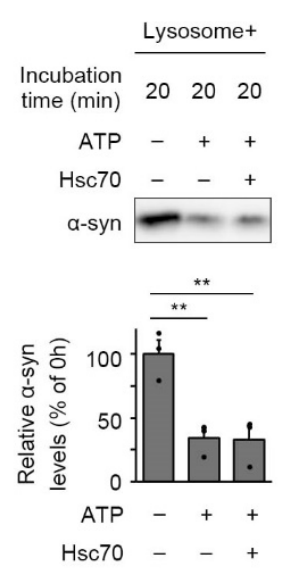

Fig. S2. Assays using isolated lysosomes derived from mouse brain tissues.

(A) Degradation of $\alpha$-synuclein protein by isolated lysosomes derived from mouse brain $(\mathrm{n}=3)$. pre-treated in the absence or presence of $\operatorname{ATP}(n=3)$. (C) Levels of $\alpha$-synuclein protein in the pellet of isolated lysosomes and external solution following co-incubation with the $\alpha$-synuclein protein $(\mathrm{n}=3)$. (D) Degradation of recombinant mutant $\alpha$-synuclein protein lacking the KFERQ-like motif by isolated lysosomes $(\mathrm{n}=3)$. (E) Degradation of recombinant WT $\alpha$-synuclein protein by isolated lysosomes with or without Hsc70 chaperone $(n=3) . * * p<0.001$. 
A

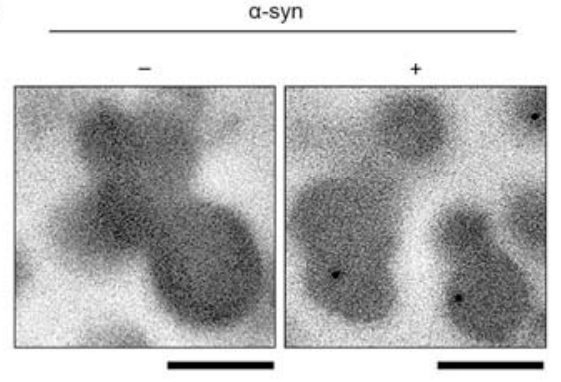

B

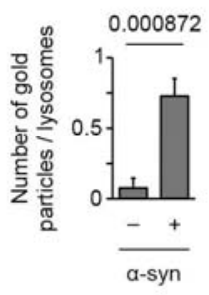

C

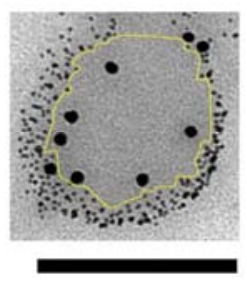

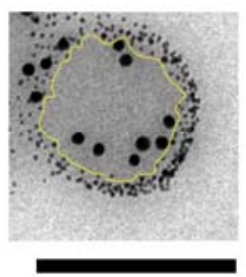

Fig. S3. Immunoelectron microscopic observations of $\alpha$-synuclein protein uptake into lysosomes.

(A to C) Post-embedded immunoelectron microscopy image of $\alpha$-synuclein protein in isolated lysosomes co-incubated with (A to $\mathrm{C}$ ) or without (A) recombinant $\alpha$-synuclein protein in the presence of ATP. Bars represent $200 \mathrm{~nm}$. First, $5 \mathrm{~nm}$ gold particles were co-incubated with lysosomes and $\alpha$-synuclein protein in the presence of ATP, and this was followed by immunoelectron microscopy against $\alpha$-synuclein protein using $10 \mathrm{~nm}$ gold particles (B and C). While $5 \mathrm{~nm}$ gold particles were adsorbed on the surface of lysosomes, $15 \mathrm{~nm}$ gold particles indicating $\alpha$-synuclein protein were seen in the luminal area of the lysosomes. The yellow line indicates the approximate delimitation between the inside and outside of lysosomes. 
A
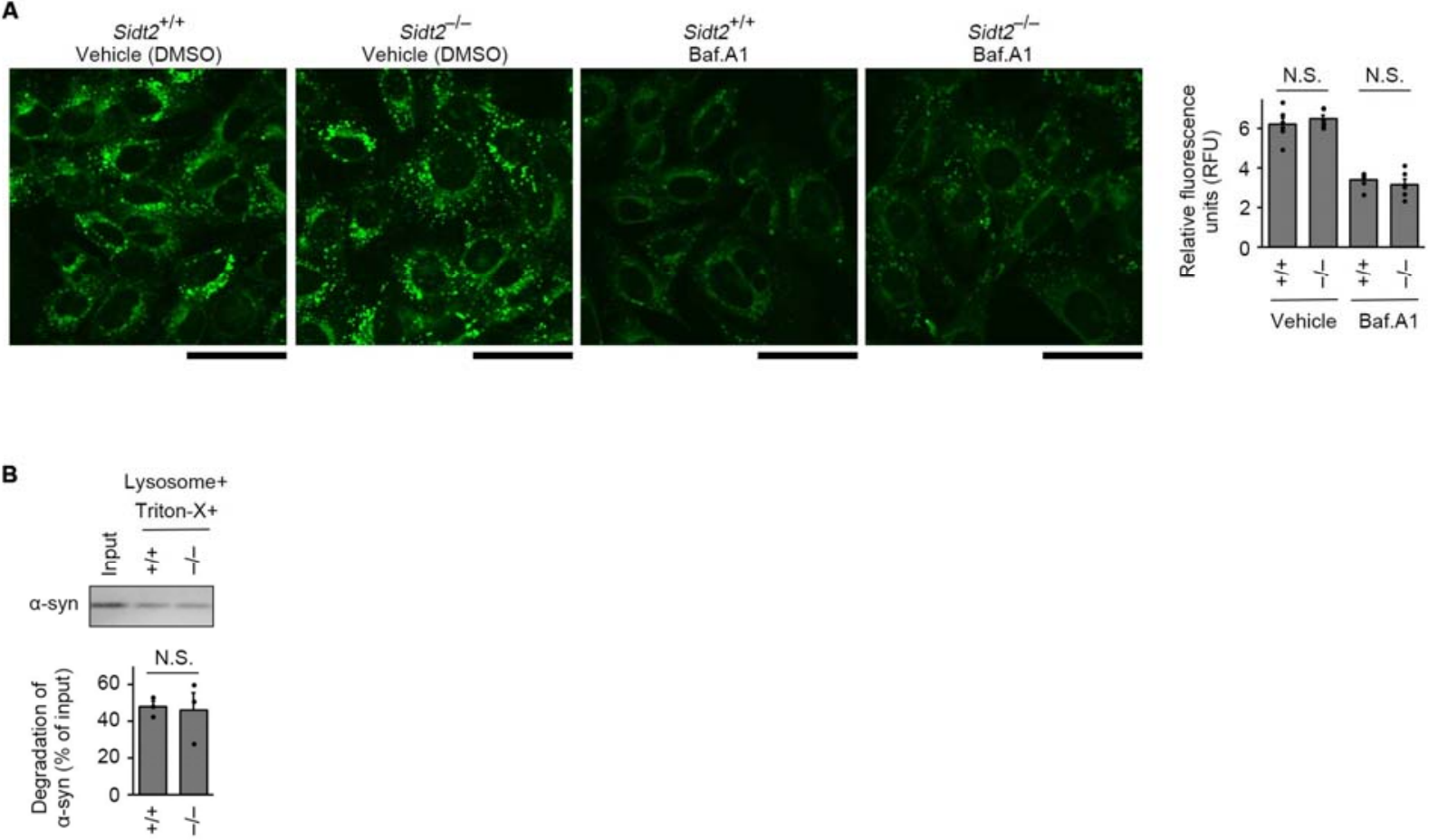

Fig. S4. SIDT2 does not affect the intra-lysosomal proteolytic capacity, and protein degradation by isolated lysosomes takes place inside the lumen of lysosomes.

(A) Acidity of lysosomes in Sidt2 ${ }^{+/+}$and Sidt $2^{-/}$MEFs treated with bafilomycin A1 or DMSO (n = 6). Bars represent $50 \mu \mathrm{m}$. (B) Degradation of $\alpha$-synuclein protein incubated with dissolved isolated lysosomes derived from Sidt2 $2^{+/+}$or Sidt $2^{-/-}$MEFs $(\mathrm{n}=3)$. 
A

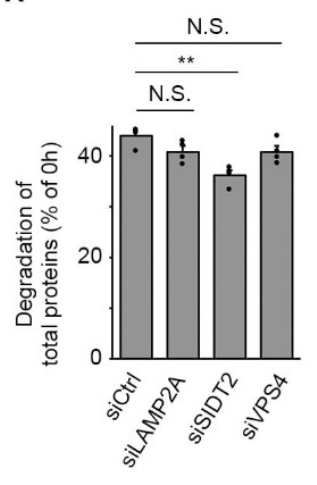

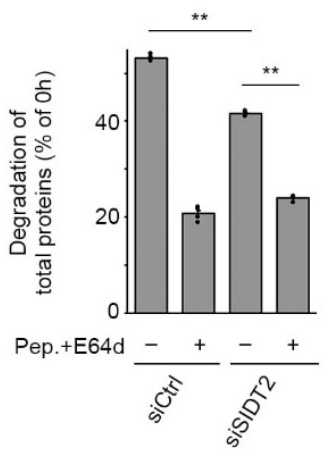

C

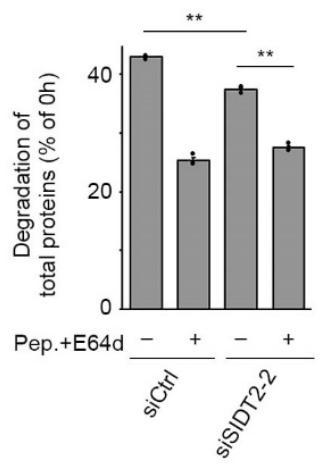

D
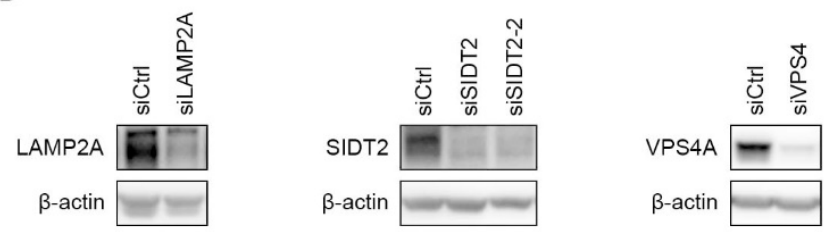

Fig. S5. Contribution of SIDT2 and other genes related to canonical types of autophagy on steady-state levels of proteolysis.

(A) Effect of Lamp2A, Sidt2, or Vps4a and $b$ knockdown on degradation of radiolabeled total proteins in $\operatorname{Atg} 5^{--}$MEFs $(\mathrm{n}=4)$. ( $\mathbf{B}$ and $\left.\mathbf{C}\right)$ Effect of Sidt2 knockdown and lysosomal protease inhibitors on degradation of radiolabeled total proteins in $A \operatorname{tg} 5^{-1-} \mathrm{MEFs}$, using two different siRNAs against SIDT2 $(\mathrm{n}=4)$. (D) Expression levels of LAMP2A, SIDT2, and VPS4A in Atg $5^{-1-}$ MEFs knocked down for respective gene products. ** $\mathrm{p}<0.001$. 
A
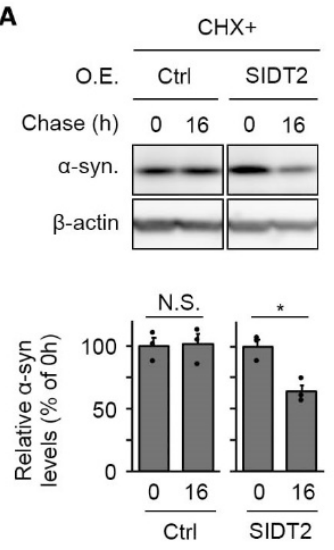

B
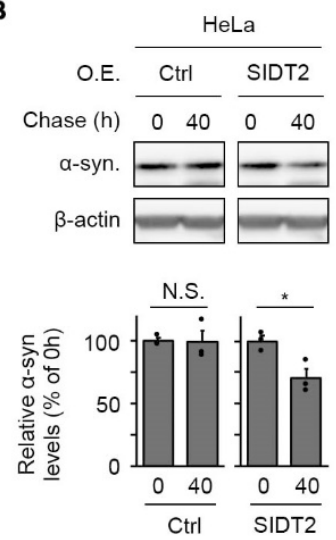

C
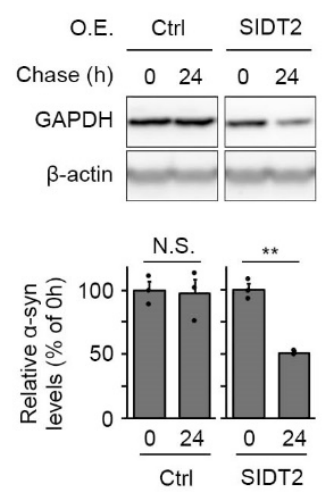

D
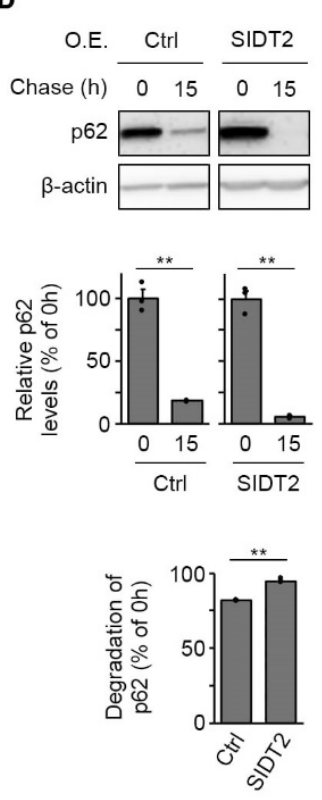

E
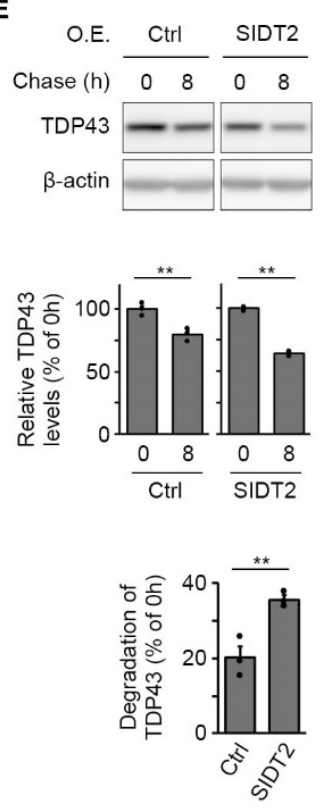

$\mathbf{F}$
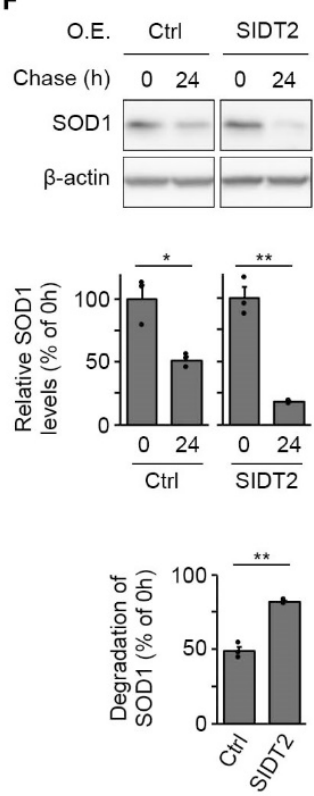

G
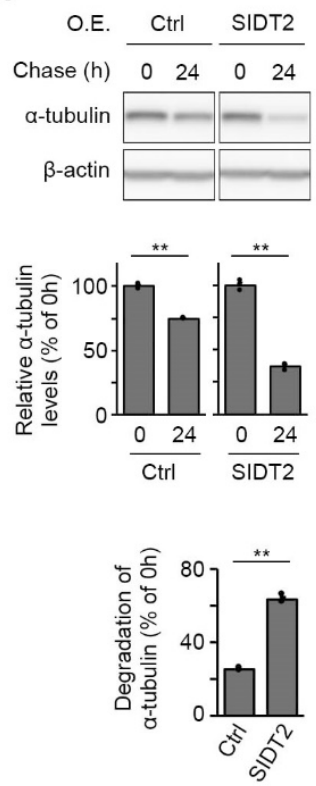

Fig. S6. SIDT2 mediates lysosomal degradation of various proteins.

(A) Degradation of $\alpha$-synuclein protein in N2A cells overexpressing SIDT2 using the Tet-Off

system, under cycloheximide treatment to additionally inhibit protein synthesis during the chase $(\mathrm{n}=3)$. (B) Degradation of $\alpha$-synuclein protein in HeLa cells overexpressing SIDT2, using the Tet-Off system (n = 3). (C to G) Degradation of GAPDH (C), p62 (D), TDP43 (E), SOD1 (F), and $\alpha$-tubulin (G) proteins in N2A cells overexpressing SIDT2, using the Tet-Off system $(\mathrm{n}=3)$. ${ }^{*} \mathrm{p}<0.05, * * \mathrm{p}<0.001$ 
A

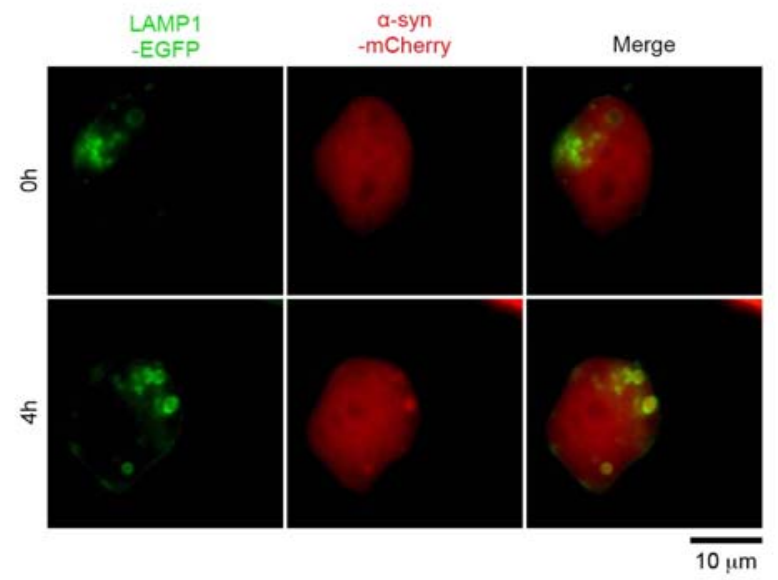

B

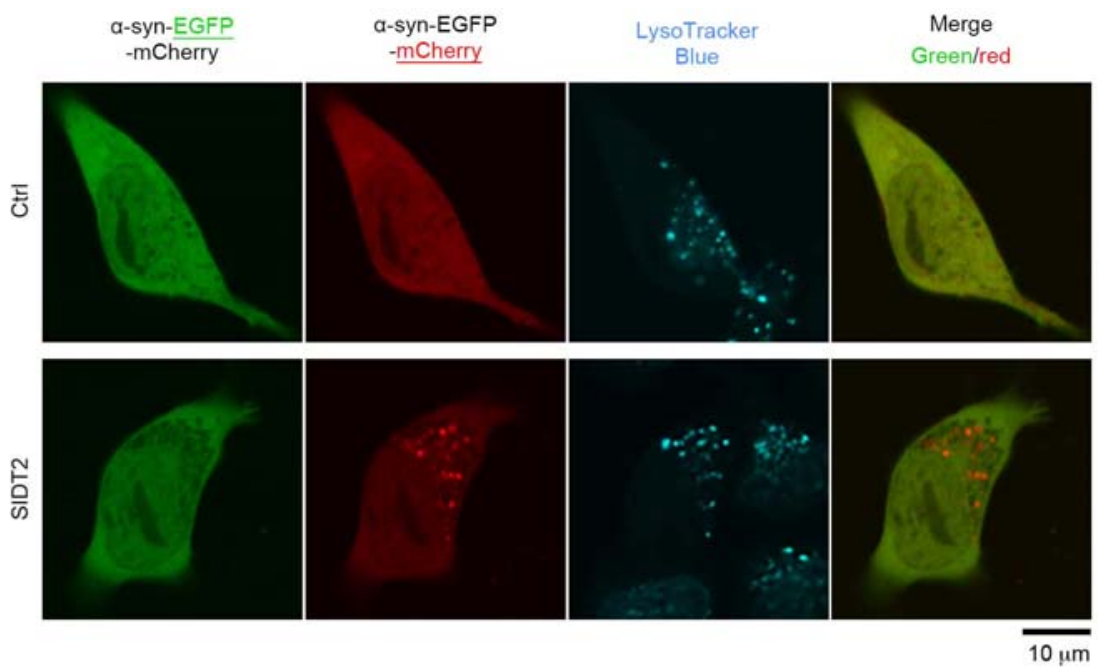

Fig. S7. Localization of fluorescent protein-labelled $\alpha$-synuclein protein in

\section{SIDT2-overexpressing cells.}

(A) Time-lapse imaging of $\alpha$-synuclein-mCherry in an N2A cell overexpressing SIDT2.

$\alpha$-synuclein-mCherry is primarily distributed in a diffuse state, and then subsequently

translocates to lysosomes. (B) Left panel: Schematic of the $\alpha$-synuclein-EGFP-mCherry

construct. When $\alpha$-synuclein-EGFP-mCherry is transported into acidic organelles such as

lysosomes, the EGFP signal is selectively quenched, leaving behind mCherry-positive only 
puncta. Right panel: Localization of $\alpha$-synuclein-EGFP-mCherry protein in

SIDT2-overexpressing N2A cells. EGFP-negative but mCherry-positive puncta that localize to lysosomes indicate that the protein is imported into the lumen of lysosomes. 
A

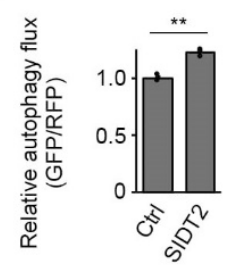

B

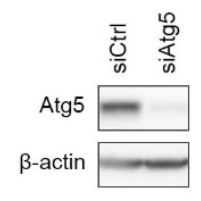

C
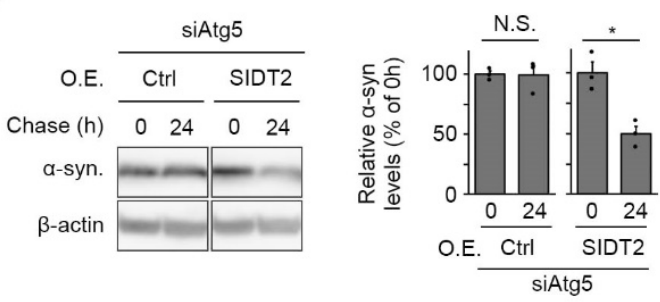

D

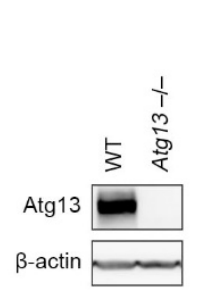

E

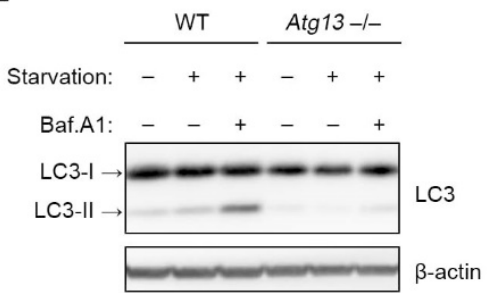

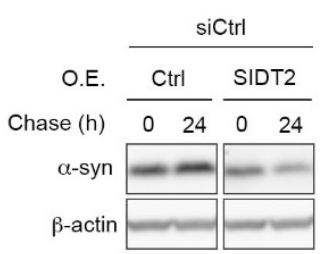

$\mathrm{F}$

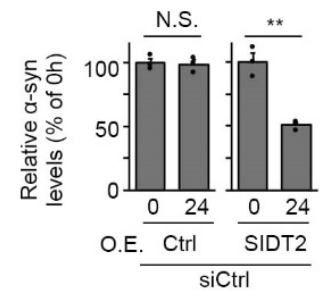

G

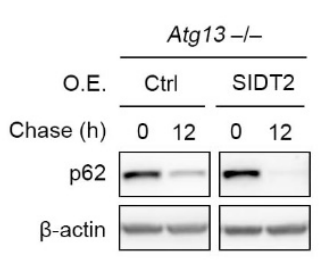

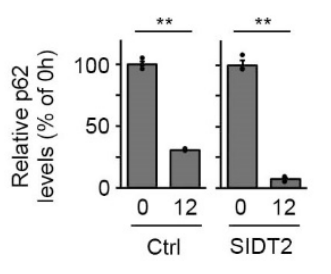

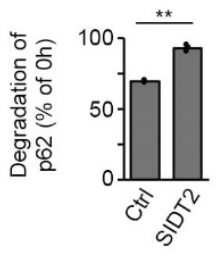

$\mathrm{H}$
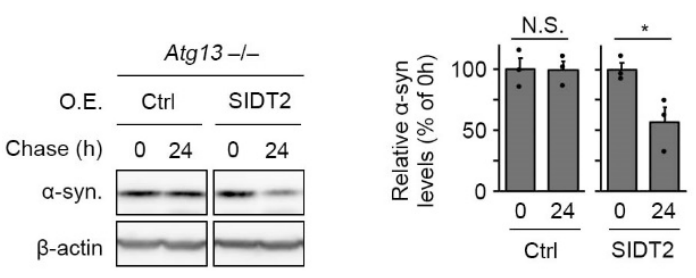
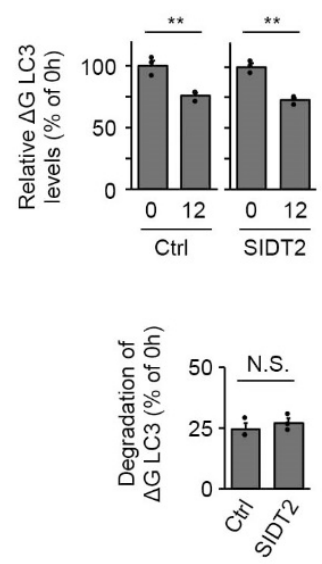

\section{Fig. S8. SIDT2-dependent proteolysis is independent of macroautophagy.}

(A) Autophagic flux in N2A cells overexpressing SIDT2 $(n=3)$. The higher GFP/RFP ratio 
cells treated with siRNA against Atg5 or control siRNA ( $=3)$. (D) Immunoblotting of Atg13 in Atg13 KO N2A cells. (E) Immunoblotting of LC3 in WT and Atg13 KO N2A cells cultured in normal or starvation medium with or without bafilomycin A1, indicating that macroautophagy is abrogated in the Atg13 KO cells. (F to H) Degradation levels of $\alpha$-synuclein (F), p62 (G), and $\Delta \mathrm{G}$ LC3, a mutant LC3 with a deletion of the C-terminal glycine (the mutant does not convert to LC3-II), (H) proteins in SIDT2-overexpressing Atg13 KO N2A cells ( $\mathrm{n}=3) . * \mathrm{p}<0.05, * * \mathrm{p}<$ 0.001 . 
A
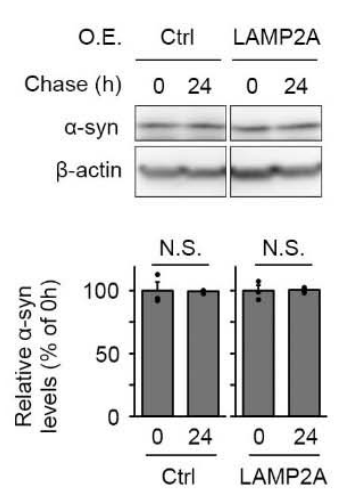

B
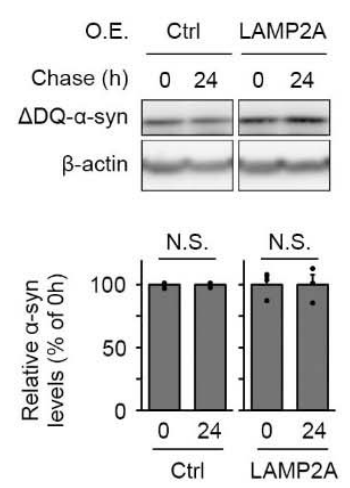

Fig. S9. LAMP2A does not accelerate proteolysis in N2A cells.

(A) Degradation of $\alpha$-synuclein protein in N2A cells overexpressing LAMP2A, using the Tet-Off system ( $\mathrm{n}=3$ ). (B) Degradation of mutant $\alpha$-synuclein protein that lacks the KFERQ-like motif in N2A cells overexpressing LAMP2A $(\mathrm{n}=3)$. 
A

Genome (Ctrl)

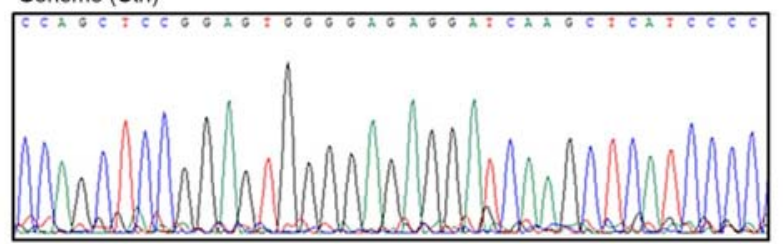

Genome (Patient)

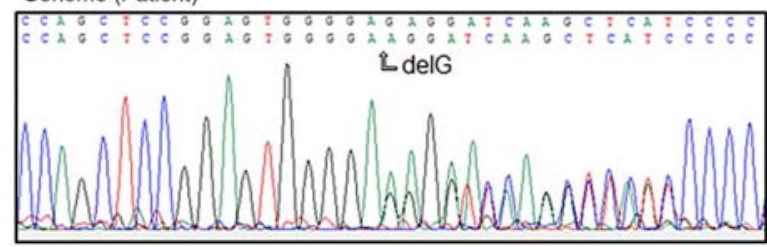

Transcript (Patient)

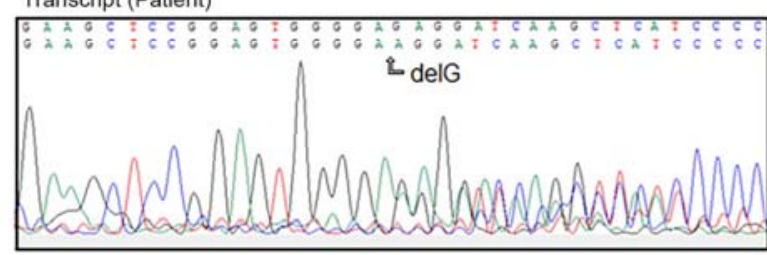

C

$$
\text { LysoTracker }
$$

$$
\text { Green }
$$

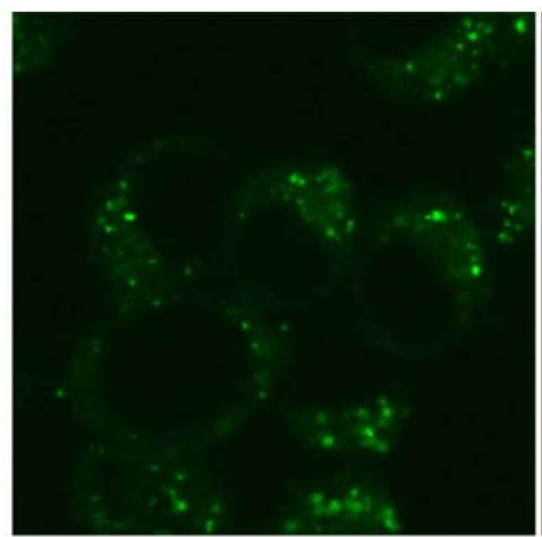

B

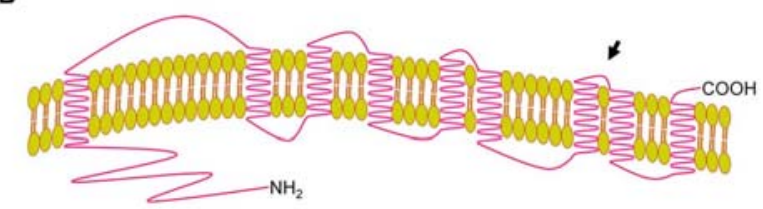

GSSSSPCSASFAPPWSGASR SSSSSRDSAPGRKPLQSRGS TTGTASSSTSLTTTTSGTSS PPSPCSGPSWCC

D

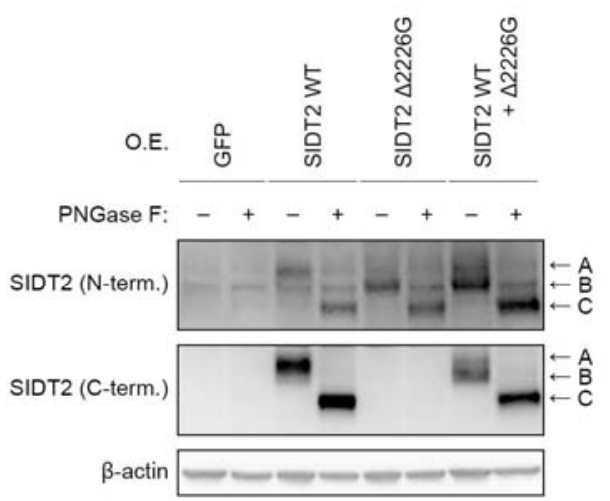

SIDT2 $\triangle 2226 \mathrm{G}$ mCherry
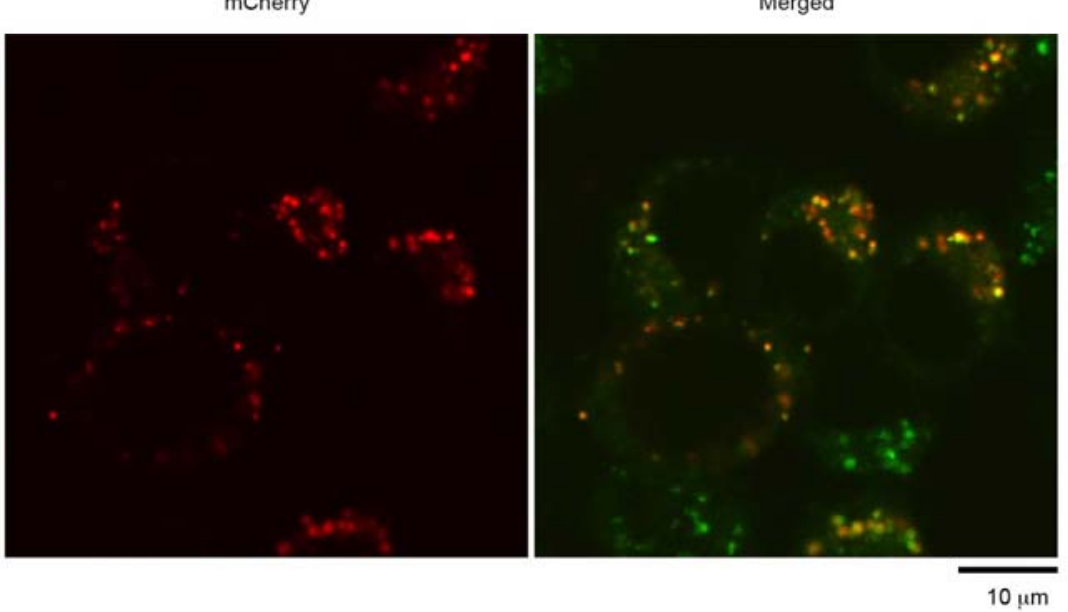

Fig. S10. Characterization of rimmed vacuolar myopathy patient harboring a $\triangle 2226 G$ mutation in SIDT2 and the mutant SIDT2.

(A) Sanger sequencing chromatogram of a control biopsy and the proband showing the heterozygous $\triangle 2226 \mathrm{G}$ frameshift mutation in SIDT2, and direct sequencing chromatogram of the 
patient's SIDT2 transcript. This variant was not listed in any of the following public databases as of mid-March 2020: dbSNP, 1000 Genomes, gnomAD, the Human Gene Mutation Database, and ClinVar. (B) A schematic of SIDT2 protein and sequence of additional aberrant amino acid residues produced by the $\triangle 2226 \mathrm{G}$ mutation in SIDT2. The arrow indicates the site of mutation in the patient. (C) Localization of $\Delta 2226 \mathrm{G}$ SIDT2-mCherry in N2A cells. (D) Glycosylation of WT and $\triangle 2226 \mathrm{G}$ SIDT2. 

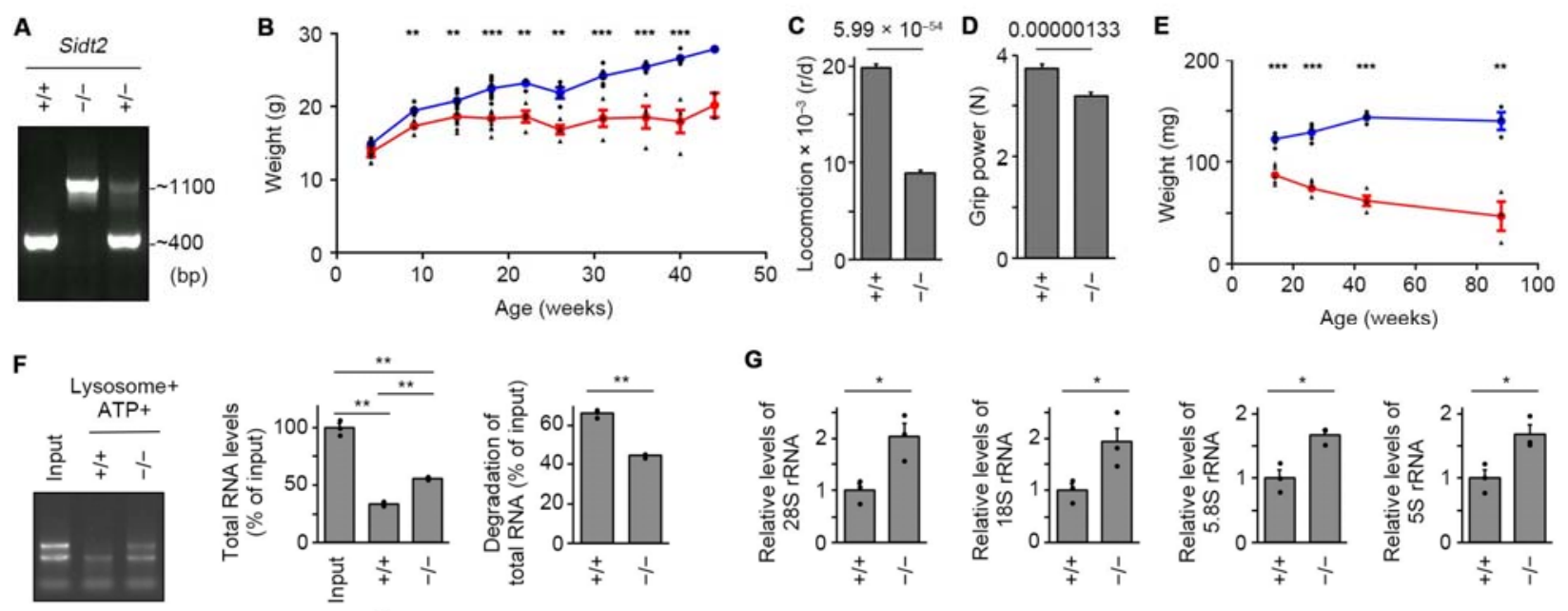

RNA
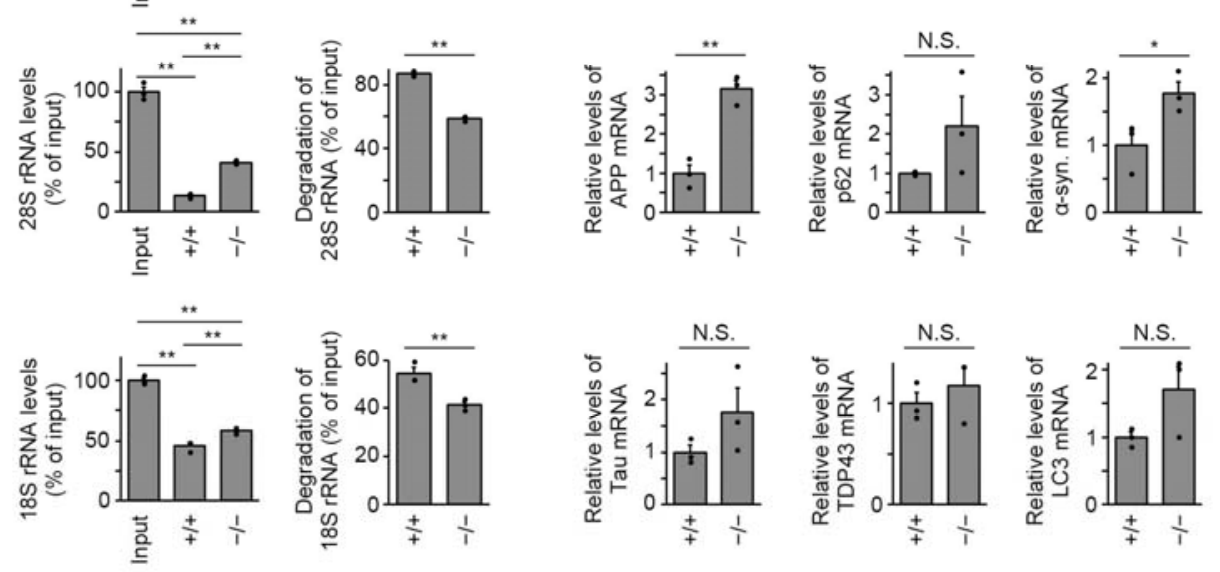

H

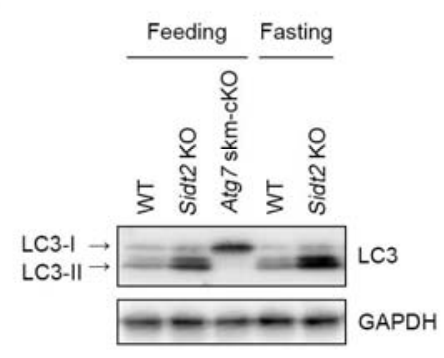

I

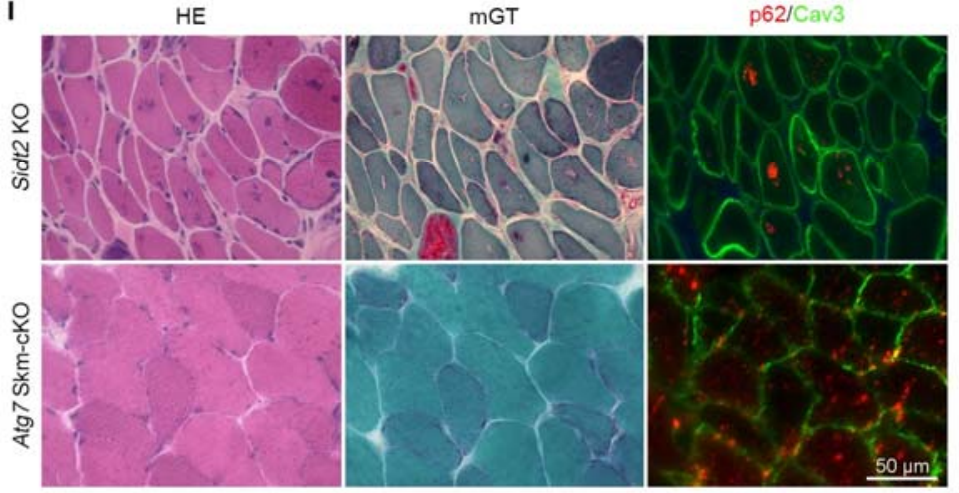

\section{Fig. S11. Characterization of Sidt2 knockout mice.}

(A) Genotyping of Sidt2 by PCR. 400 bp: WT allele-specific band, 1,100 bp: KO

allele-specific band. (B) Growth curves of Sidt $2^{+/+}$and Sidt2 $2^{-/}$mice $(\mathrm{n}=1-11)$. (C) Locomotor 
activities of $\operatorname{Sidt}^{+/+}$and Sidt $2^{-/-}$mice $\left(+/+\mathrm{n}=154\right.$ from 11 mice, ${ }^{-/} \mathrm{n}=126$ from 9 mice). (D) Grip strength of $\operatorname{Sidt}_{2}^{+/+}$and Sidt $2^{-/-}$mice $(+/+\mathrm{n}=110$ from 11 mice, $-/-\mathrm{n}=90$ from 9 mice). (E) Changes in weight of the gastrocnemius of Sidt $2^{+/+}$and Sidt $2^{-/-}$mice $(\mathrm{n}=3-5)$. (F) Degradation of RNA by isolated lysosomes derived from Sidt $2^{+/+}$or Sidt $2^{-/-}$mouse skeletal muscles $(\mathrm{n}=3)$. $(\mathbf{G})$ Levels of RNA in gastrocnemius of $\operatorname{Sidt} 2^{+/+}$or $\operatorname{Sidt} 2^{-/-}$mice $(\mathrm{n}=3)$. $(\mathbf{H})$ Immunoblotting of LC3 in feeding and fasting Sidt $2^{+/+}$and Sidt $2^{-/-}$mouse skeletal muscles. (I) Comparison of skeletal muscle pathology of Sidt $2^{-/-}$mice and Atg7 skeletal muscle conditional KO mice. Although atrophy is also occurring, rimmed vacuoles were not observed, and p62 deposits were more diffusely observed in the macroautophagy-deficient muscles, compared with the level in Sidt $2^{-/}$muscles. $* \mathrm{p}<0.05, * * \mathrm{p}<0.001, * * * \mathrm{p}<0.0001$. 


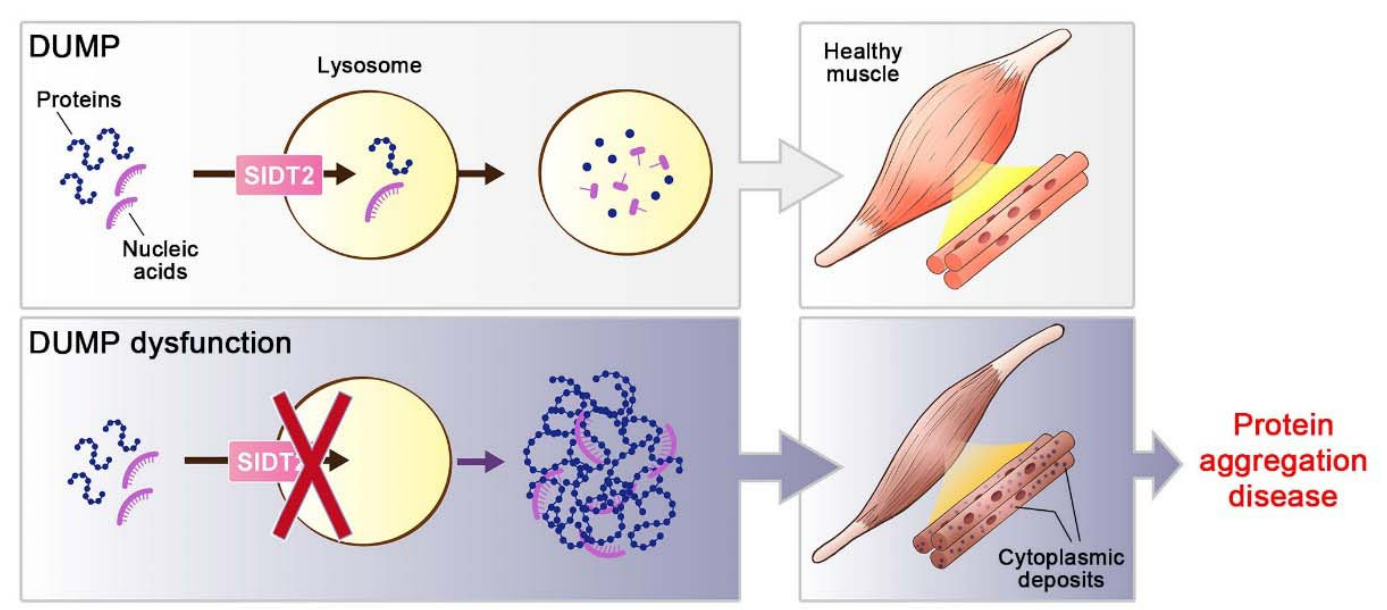

\section{Fig. S12. A schematic of DUMP dysfunction leading to protein aggregation disease.}

The process of DUMP maintains homeostasis in vivo (upper panel). Impairment of DUMP caused by mutation or deficiency in SIDT2 results in an accumulation of intracellular deposits in the cytoplasm and induces neuromuscular dysfunction, leading to neuropathy and myopathy with rimmed vacuoles (lower panel). 